\title{
Diversity and Distribution Patterns of Endemic Medicinal and Aromatic Plants of Iran: Implications for Conservation and Habitat Management
}

\author{
Mohammad Bagher Hassanpouraghdam ${ }^{1, *(1)}$, Hamideh Ghorbani ${ }^{1}$, Marzieh Esmaeilpour ${ }^{2}$, Mac H. Alford ${ }^{3}$, \\ Maciej Strzemski ${ }^{4}\left(\mathbb{D}\right.$ and Sławomir Dresler ${ }^{4,5} \oplus$
}

check for

updates

Citation: Hassanpouraghdam, M.B.; Ghorbani, H.; Esmaeilpour, M.;

Alford, M.H.; Strzemski, M.; Dresler, S. Diversity and Distribution Patterns of Endemic Medicinal and Aromatic Plants of Iran: Implications for Conservation and Habitat Management. Int. J. Environ. Res. Public Health 2022, 19, 1552. https:// doi.org/10.3390/ijerph19031552

Academic Editor: Paul B. Tchounwou

Received: 30 December 2021

Accepted: 27 January 2022

Published: 29 January 2022

Publisher's Note: MDPI stays neutral with regard to jurisdictional claims in published maps and institutional affiliations.

Copyright: (C) 2022 by the authors. Licensee MDPI, Basel, Switzerland. This article is an open access article distributed under the terms and conditions of the Creative Commons Attribution (CC BY) license (https:/ / creativecommons.org/licenses/by/ $4.0 /)$.
1 Department of Horticultural Science, Faculty of Agriculture, University of Maragheh, Maragheh 55181-83111, Iran; h_ghorbani30@yahoo.com

2 Department of Geography, University of Maragheh, Maragheh 55181-83111, Iran; m.esmaeilpour@maragheh.ac.ir

3 School of Biological, Environmental, and Earth Sciences, University of Southern Mississippi, Hattiesburg, MS 39406, USA; mac.alford@usm.edu

4 Department of Analytical Chemistry, Medical University of Lublin, 20-093 Lublin, Poland; maciej.strzemski@poczta.onet.pl (M.S.); slawomir.dresler@umlub.pl (S.D.)

5 Department of Plant Physiology and Biophysics, Institute of Biological Science, Maria Curie-Skłodowska University, Akademicka 19, 20-033 Lublin, Poland

* Correspondence: hassanpouraghdam@gmail.com; Tel.: +98-91-4502-7100

\begin{abstract}
Iran, with its unique climatic and topographic conditions, is home to about 8200 species of vascular plants. Approximately 2300 of the 8200 species are popularly characterized as medicinal or aromatic. Here, we compile information about the endemic medicinal and aromatic plants (MAPs) of Iran and map their distributions. Our survey found 180 endemic species of MAPs, belonging to 10 families and 30 genera. The majority of species are found in Lamiaceae, Fabaceae, and Apiaceae, with 86,30 , and 18 species, respectively. Approximately $70 \%$ of these plants have been recorded in the 10 provinces of Esfahan, Kerman, Fars, Tehran, Chaharmahal va Bakhtiari, East Azarbaijan, Lorestan, West Azarbaijan, Hamadan, and Mazandaran. These provinces are located in the Iran-o-Turanian region, one of the three major phytogeographic regions in Iran, which covers five areas of endemism (i.e., Azarbaijan, Zagros, Kopet Dagh-Khorassan, Alborz, and Central Alborz). So, Iran-o-Turanian region is the main center of diversity for the Iranian endemic MAPs. The north, center and western parts of Iran are rich in MAPs and could be considered as the dominant biodiversity hotspots of Iran more seemingly due to the diverse climatic and geographic assortment which generates the highest frequency and distribution of MAPs. Many of these MAPs are at the edge of extinction due to the unwise, unscientific harvesting and/or global climate change. Therefore, there is an urgent need to conserve and propagate some of these important MAPs to save them from extinction and also to ensure the availability of raw materials for their use and future research into their efficacy. Furthermore, identifying the areas of endemism (AEs) is an essential part of ongoing regional conservation management programs in Iran and worldwide.
\end{abstract}

Keywords: biodiversity hotspots (BHs); Irano-Anatolian Hotspot; medicinal and aromatic plants (MAPs); endemism; extinction; conservation

\section{Introduction}

Endemic plant species are plants that exist in one particular geographical region and nowhere else on the globe [1], and endemism is the status of being endemic or being restricted to a distinct geographical district [2]. The geographical region can be as small as an island or as large as a continent. Areas with high concentrations of endemic species and with significant habitat loss are also referred to as "Biodiversity Hotspots" (BHs) [3]. Currently, 36 areas around the world are considered BHs [2]. 
The fundamental hazard of endemic species is that they are more susceptible to extinction. Since they are limited geographically, the devastation of their habitat due to man-made enterprises and/or global climate change could reduce their populations drastically [4]. Because endemism is deemed as a substantial factor for biodiversity conservation at the local, national, and global scale, identifying the number and distribution of endemic plants in a biogeographic area is a preliminary point for evaluating the protection of that defined region [5].

Of the 422,000 species of vascular plants in the world, 50,000-80,000 of them are used medicinally and contain valuable ethnobotanical and remedial information that could navigate new drug exploration [6,7]. As one of the significant bio-resource centers of the world, the Asian continent accounts for over 38,660 species of MAPs (Medicinal and Aromatic Plants) [8]. Nowadays, the application of MAPs is increasing due to their rich capacity for the treatment of diverse maladies and their fewer side effects [9]. Therefore, the tendency in worldwide research has focused more on the search for new medicines and active compounds of MAPs rather than on the cultivation and/or domestication of the plant species with this characteristic potential [10]. Increasing population pressure, overharvesting, unscientific collection by untrained persons, excessive grazing, fire, and global climate change have placed many of these plants at the risk of extinction [11]. Experts estimate that the Earth is losing at least one potential primary drug every two years [6].

Setting priorities is necessary for the conservation management of MAPs. So, identifying BHs in the world and mapping the AEs (Areas of Endemism)of every country can be used as a powerful method for the prioritization of the endemic MAPs conservation [3]. A total of $36 \mathrm{BHs}$ have been designated, covering 16.7\% of Earth's land surface and home to $77 \%$ of all endemic plant species [2]. For conservation management purposes, however, they are practically too large, so an appropriate method to achieve protection of these species is the identification and studying of the AEs within a global biodiversity hotspot [1,12].

One of the global BHs located entirely within southwest Asia is the Irano-Anatolian hotspot, which extends over an area of about $899,773 \mathrm{~km}^{2}$ and is home to about 6000 plant species [13]. Iran covers an estimated 54\% of the surface area of the IranoAnatolian hotspot [14]. Another BH, the Caucasus, includes Georgia, Azarbaijan, and a small portion of northern Iran (around 10\%) [1,12]. So, Iran is home to two of the world's BHs: The Irano-Anatolian and Caucasus.

Iran is a vast country $\left(1,648,195 \mathrm{~km}^{2}\right)$, with different climates ranging from mainly arid to semi-arid and also mountainous [15], and is at the intersection of three well-known phytogeographic areas (the Iran-o-Turanian, the Saharo-Sindian, and the Euro-Siberian) [16]. The majority of Iran is located in the Iran-o-Turanian region and is divided into two subregions: mountainous areas and an area of high plains and deserts [15]. The driest portion of Iran-o-Turanian (desert sub-region) is dominated by arid and hyper-arid climates and has reasonable plant diversity.

It seems that the environmental and climatic features have had a fundamental influence on the endemic diversity and richness of Iran. The dominant climates of Iran include arid, semi-arid, hyper-arid as well as small territories of humid, semi-humid, highly-humid, and Mediterranean [17].

According to Noroozi et al. (2019), the Iran-o-Turanian region harbors about $88 \%$ of the Iranian endemic species. Given the climatic conditions and diversity of endemic species, this region has been further subdivided into five AEs: Alborz, Central Alborz, Zagros, Azarbaijan, and Kopet Dagh-Khorasan [1,12,14].

The Alborz and Zagros Mountains expand in a northwest-northeast and northwestsoutheast orientation [18] and are situated in East and West Azarbaijan, Tehran, Kurdistan, Kermanshah, Lorestan, Fars, Chaharmahal va Bakhtiari, Esfahan, Yasouj, Markazi, and the northern part of Khozestan Provinces. Other significant AEs are Iranian Azarbaijan in the northwest and Kopet Dagh-Khorasan that stretches from the eastern boundary of the Caspian Sea into northeastern Iran [19]. 
The spectacular nature of this Iranian plateau with five AEs, is full of several species of endemic MAPs with important potential pharmaceutical and therapeutic properties that might not have been widely reported or thoroughly studied to date. People have long occupied this area, with an early center of civilization that dates back to the BabylonianAssyrian era [20]. One of the most considerable parts of this ancient heritage is the science of people who figured out helpful plants for health improvement, with subsequent generations appending their own experience and skill to this custom [20].

Approximately 8200 vascular plant species are recognized in Iran, of which almost 2300 are medicinal and aromatic $(28 \%)$ [13,21,22]. Among these are numerous endemic species that are only known from Iran. Many studies have been performed on species of endemic MAPs based on chemical composition and biological activity, but little attention has been paid to the distribution patterns, areas of endemism, and conservation zones of endemic MAPs in Iran.

In this review of the endemic MAPs of Iran, we compiled and analyzed data with the following aims: (1) to determine the families and genera with high endemic richness, (2) to determine the provinces with higher endemic species richness, (3) to recognize the distribution patterns and areas of endemism for medicinal and aromatic species of the country, and (4) to present some suggestions for conservation of these areas of endemism.

\section{Methods}

We reviewed scientific studies published in reliable journals and books. Pertinent literature was searched for in electronic databases such as Magiran, Scopus, Google Scholar, Web of Science, Science Direct, and PubMed and books (including some dictionaries of Iranian plants compiled by Valiallah Mozafarian and Ahmad Ghahreman [23], and the Red Data Book of Iran: A Preliminary Survey of Endemic, Rare \& Endangered Plant Species in Iran, compiled by Adel Jalili and Ziba Jamzad) [24], using specific search terms such as biodiversity hotspots, Irano-Anatolian hotspot, medicinal and aromatic plants, medicinal herbs, traditional plants, endemism, extinction, and conservation.

We do not claim to have included every source about endemic MAPs of Iran, rather we focused on data available on the internet and in libraries that are accessible to scholars. We found and reviewed a total of 165 articles that provided details about endemic Iranian MAPs that are used to treat various ailments and disorders.

From these resources, we compiled a list of endemic MAPs, showing family, scientific names, common names, parts used, therapeutic effects and ethnopharmacological properties, provinces (habitats), and references for each species.

The distribution maps were prepared using a geographic information system (ArcGis 10.2.2), with the country was divided into three major phytogeographic districts (the Irano-Turanian, the Saharo-Sindian, and the Euro-Siberian) and five AEs (Alborz, Central Alborz, Zagros, Azarbaijan, and Kopet Dagh-Khorasan) according to the distribution of the endemic MAPs across provinces of Iran. A distribution map of endemic MAPs by different climatic conditions of Iran was also drawn.

\section{Results and Discussion}

\subsection{Taxonomic Divisions of Endemic MAPs in Iran}

Vascular plants of Iran comprise 65 families, 359 genera, and about 8200 species, of which 2597 of them are endemic (32\% of all native species) [1,12]. Of these, we found that 180 endemic MAPs, which belong to 30 genera and 10 families, are used in Iran. The 10 families in terms of the number of endemic MAPs are Lamiaceae (84 spp.), Apiaceae (37 spp.), Fabaceae (21 spp.), Asteraceae (7 spp.), Hypericaceae (2 spp.), Alliaceae (2 spp.), Rosaceae (3 spp.), Boraginaceae (3 spp.), Scrophulariaceae (3 spp.), and Liliaceae (3 spp.) (Figure 1). A total of 10 families are representing the major medicinal plants of Iran with about 165 most dominant species (Figures 1 and 2 and Table 1). 


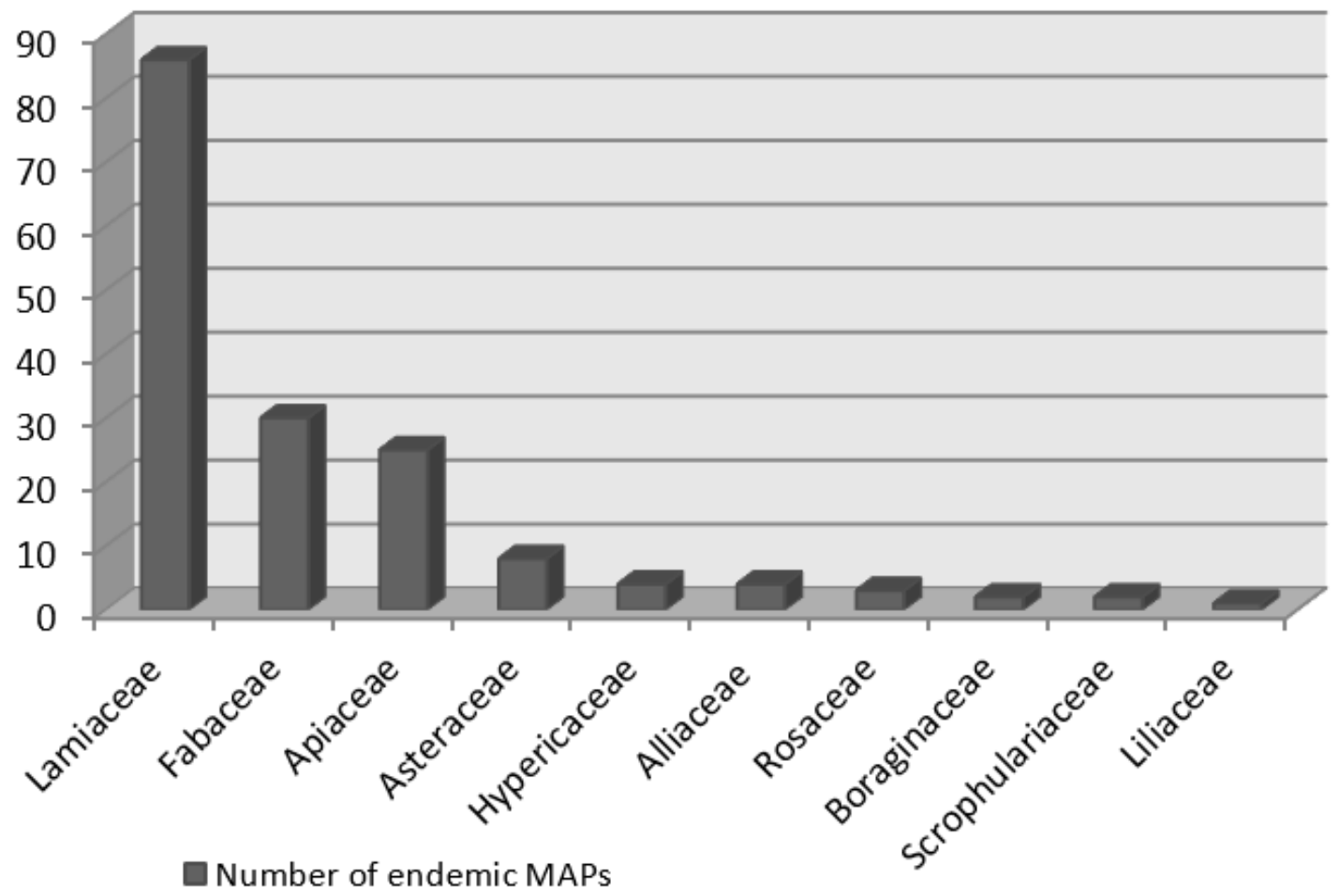

Figure 1. The species number of the ten most endemic-rich families of Iranian medicinal and aromatic plants.

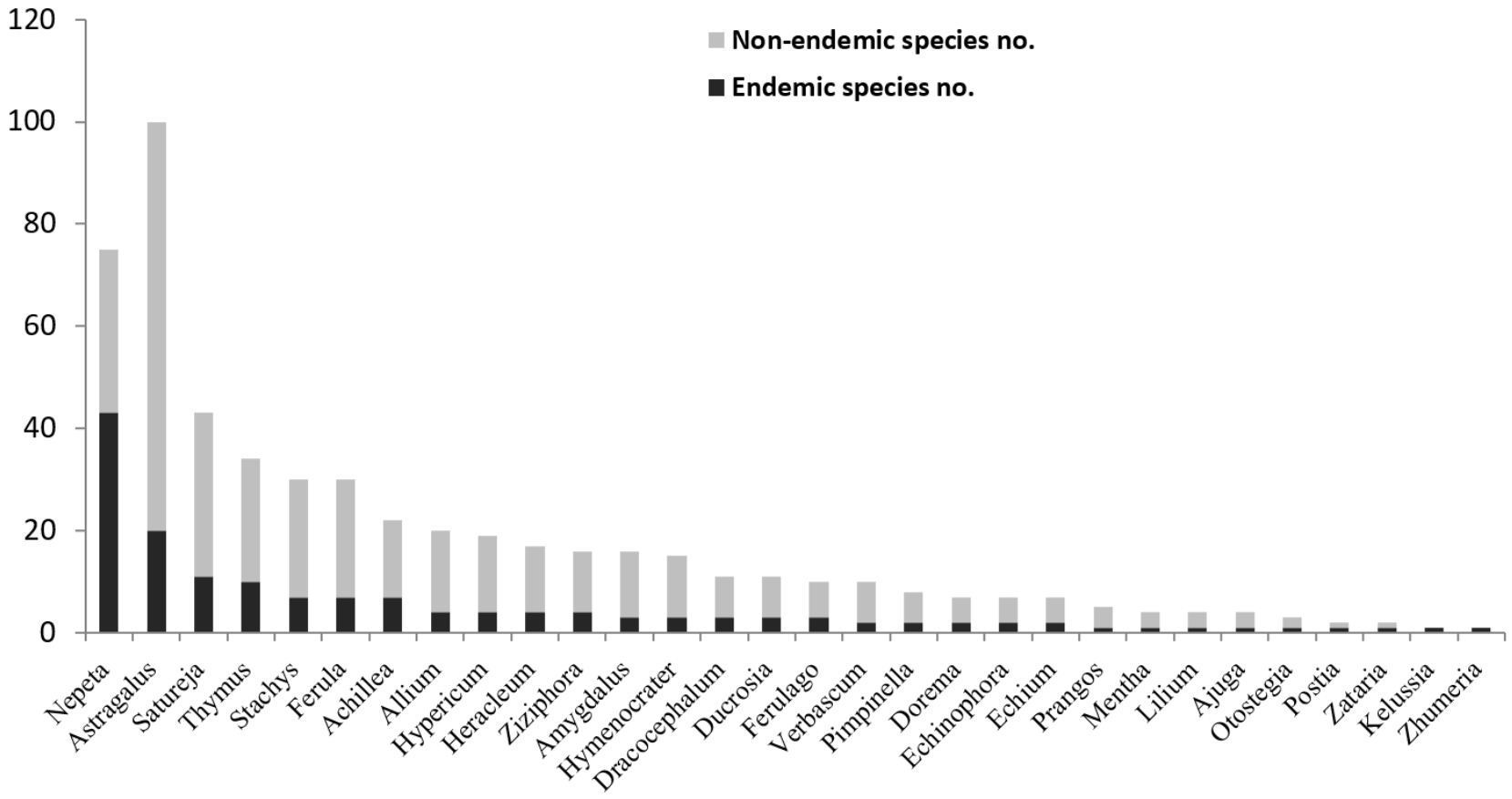

Figure 2. The frequency of endemic and non-endemic species of the 30 most endemic-rich genera of the Iranian medicinal and aromatic plants.

The largest number of Iranian endemic MAPs is observed in Lamiaceae and Fabaceae, with 86 and 30 species, respectively (Figure 1). The dominant MAPs genera of Iranian flora are presented in Figure 2 from which the notable genera include Nepeta from Lamiaceae and Astragalus from Fabaceae.

Lamiaceae is the family with the largest number of species in terms of endemic MAPs (12 genera and 86 species, Table 1), which is due to the hyper-diverse genus Nepeta 
with 43 species (53\% endemic, Figure 2). The genus Nepeta consists of about 300 species widely distributed in Europe, Asia, and some areas of Africa. Iran is one of the primary centers of this genus, with 79 species [25]. The genus Nepeta, with the common Persian name of "Pune-sa", is widely used in the folk medicine of Iran for diuretic, digestive, diaphoretic, antitussive, antispasmodic, anti-asthmatic, febrifuge, emmenagogue, and sedative effects $[25,26]$.

The second most prominent family in terms of endemic MAPs is Fabaceae (Figure 1), with most species in the genus Astragalus. Iran is one of the world's centers of Astragalus diversity, which makes up $21 \%$ of Iran's endemic vascular plants. Furthermore, Fabaceae is the second largest family of Iranian flora containing a large number of native species (850 species) and the total number of endemic species (527 species), but we found only 30 endemic MAPs (Figure 2, Table 1). Meanwhile, the interest in the chemical compounds of different species of Astragalus has been increasing; these data may change as the new endemic MAPs species continue to be characterized [15].

Other families with high numbers of endemic MAPs are Apiaceae, Asteraceae, Rosaceae, Boraginaceae, Hypericaceae, Liliaceae, Scrophulariaceae, and Alliaceae. As species conservation and taxonomy are often assumed to be interdependent activities, it is suggested that these families should be further studied, especially their medicinal and aromatic species, since a shortage of taxonomic information can cause problems for conservationists [13].

In addition to the traditional and newly characterized medicinal plants, there is another group, namely the potential medicinal plants. These kinds of plants are species that are thought to contain bioactive compounds that are medicinally efficacious. They have not been medically proven, but they are used as ingredients for traditional medicine. Table 2 presents 80 endemic MAPs of Iran which have only been explored superficially, despite having beneficiary effects. The highlighted species need to be more thoroughly studied for their active ingredients to justify the traditional usage of the species and to aid the search for components that may be used in modern medicine [27-30].

Table 1. List of endemic medicinal and aromatic plants identified from Iran.

\begin{tabular}{|c|c|c|c|c|c|}
\hline Scientific Name & $\begin{array}{c}\text { Common Name } \\
\text { (Vernacular Name) }\end{array}$ & Parts Used & $\begin{array}{l}\text { Therapeutic Effects \& Ethno } \\
\text { Pharmacological Properties } \\
\text { (The Number Inside } \\
\text { Paranthesis of Families Refers } \\
\text { to the Number of } \\
\text { Endemic Species) }\end{array}$ & Province(s) & Ref. \\
\hline \multicolumn{6}{|c|}{ Alliaceae (2) } \\
\hline $\begin{array}{l}\text { Allium hirtifolium } \\
\text { Boiss. }\end{array}$ & Mosir & bulb & $\begin{array}{l}\text { antioxidant, hypertension, } \\
\text { rheumatoid, inflammation, } \\
\text { wounds healing, antibacterial, } \\
\text { antifungal, and anticancer }\end{array}$ & $\begin{array}{c}\text { West Azarbaijan, } \\
\text { Kurdestan, } \\
\text { Kermanshah, } \\
\text { Hamadan, Lorestan, } \\
\text { Esfahan, } \\
\text { Chaharmahal va } \\
\text { Bakhtiari, Kohkiluyeh } \\
\text { va Boyer-Ahmad, Fars, } \\
\text { Arak, Yasouj, }\end{array}$ & {$[31,32]$} \\
\hline $\begin{array}{l}\text { Allium jesdianum } \\
\text { Boiss. \& Buhse }\end{array}$ & $\begin{array}{l}\text { Bon-e-Sorkh } \\
\text { or Lizak }\end{array}$ & aerial parts & $\begin{array}{l}\text { antifungal exhibits cytostatic } \\
\text { and cytotoxic activities against } \\
\text { several malignant tumor cells }\end{array}$ & $\begin{array}{l}\text { Chaharmahal va } \\
\text { Bakhtiari, Lorestan }\end{array}$ & [33] \\
\hline \multicolumn{6}{|c|}{ Apiaceae (18) } \\
\hline $\begin{array}{l}\text { Dorema } \\
\text { ammoniacum } \\
\text { D. Don. }\end{array}$ & $\begin{array}{l}\text { Kandal, Vasha and } \\
\text { Koma-kandal }\end{array}$ & $\begin{array}{l}\text { ripe fruit, stem, leaf } \\
\text { root, and flower }\end{array}$ & $\begin{array}{l}\text { carminative, diaphoretic, mild } \\
\text { diuretic, expectorant, } \\
\text { antimicrobial, and } \\
\text { vasodilator agent }\end{array}$ & $\begin{array}{c}\text { Yazd, Esfahan, Semnan, } \\
\text { Fars, Sistan va } \\
\text { Baluchestan, Tehran }\end{array}$ & [34] \\
\hline $\begin{array}{c}\text { Dorema aucheri } \\
\text { Boiss. }\end{array}$ & Kandal-e Kohi & $\begin{array}{l}\text { ripe fruit, stem, leaf } \\
\text { root, and flower }\end{array}$ & $\begin{array}{l}\text { lowering blood pressure, liver } \\
\text { injury stimulant, antispasmodic, } \\
\text { expectorant, chronic bronchitis, } \\
\text { asthma, and anti-oxidative }\end{array}$ & $\begin{array}{l}\text { Hamadan, } \\
\text { Kermanshah, Lorestan, } \\
\text { Esfahan, Fars, Kerman, } \\
\text { Semnan }\end{array}$ & [35] \\
\hline
\end{tabular}


Table 1. Cont.

\begin{tabular}{|c|c|c|c|c|c|}
\hline Scientific Name & $\begin{array}{l}\text { Common Name } \\
\text { (Vernacular Name) }\end{array}$ & Parts Used & $\begin{array}{l}\text { Therapeutic Effects \& Ethno } \\
\text { Pharmacological Properties } \\
\text { (The Number Inside } \\
\text { Paranthesis of Families Refers } \\
\text { to the Number of } \\
\text { Endemic Species) }\end{array}$ & Province(s) & Ref. \\
\hline $\begin{array}{l}\text { Ducrosia anethifolia } \\
\text { (de Candolle.) } \\
\text { Boiss. }\end{array}$ & $\begin{array}{l}\text { Moshgak and } \\
\text { Moshkbu }\end{array}$ & aerial parts & $\begin{array}{l}\text { cold remedy, cures stomach } \\
\text { hurting, sedative and painkiller } \\
\text { activity, anti-headache, back } \\
\text { pain, colic, and colds, effective } \\
\text { against insomnia and anxiety }\end{array}$ & Fars & {$[36-38]$} \\
\hline $\begin{array}{l}\text { Ducrosia assadii } \\
\text { Alava. }\end{array}$ & Moshgake bakraei & aerial parts & $\begin{array}{l}\text { anti-inflammatory, antiseptic, } \\
\text { carminative, and soporific, }\end{array}$ & Kerman & {$[39]$} \\
\hline $\begin{array}{l}\text { Ducrosia flabellifolia } \\
\text { Boiss. }\end{array}$ & $\begin{array}{l}\text { Moshgake } \\
\text { badbezani }\end{array}$ & aerial parts & antioxidant & Kerman & {$[36,40]$} \\
\hline $\begin{array}{l}\text { Echinophora cinerea } \\
\text { (Boiss.) } \\
\text { Hedge \& Lamond. }\end{array}$ & $\begin{array}{l}\text { Khosharizeh } \\
\text { Kohestani }\end{array}$ & aerial parts & antioxidant and antidiabetic & $\begin{array}{l}\text { Hamadan, Lorestan, } \\
\text { Kohkilouyeh va } \\
\text { Boyer-Ahmad, Fars, } \\
\text { Chaharmahal } \\
\text { va Bakhtiari }\end{array}$ & {$[41,42]$} \\
\hline $\begin{array}{l}\text { Echinophora } \\
\text { platyloba de } \\
\text { Candolle. }\end{array}$ & Khosharizeh & aerial parts & antimicrobial, antioxidant & $\begin{array}{c}\text { East and West } \\
\text { Azarbaijan, Kurdestan, } \\
\text { Hamadan, Lorestan, } \\
\text { Arak, Esfahan, } \\
\text { Fars, Kerman, } \\
\text { Khorasan, Tehran }\end{array}$ & {$[41,43-45]$} \\
\hline $\begin{array}{l}\text { Ferula assa-foetida } \\
\text { Linnaeus. }\end{array}$ & Anghoseh & gum, resin & $\begin{array}{l}\text { antispasmodic, aromatic, } \\
\text { carminative, digestive, } \\
\text { expectorant, laxative, sedative, } \\
\text { analgesic, anthelmintic, } \\
\text { aphrodisiac, anticonvulsant, } \\
\text { diuretic, tonic, and antiseptic }\end{array}$ & Kerman, Khorasan & {$[46,47]$} \\
\hline Ferula persica Boiss. & Koma & aerial parts, root & $\begin{array}{l}\text { anti-pigmentation in Serratia } \\
\text { marcescens, cytotoxic, } \\
\text { antibacterial, anti-fungal, cancer } \\
\text { chemo-preventive, reversal of } \\
\text { multi-drug resistance, } \\
\text { anti-inflammatory, } \\
\text { lipoxygenase inhibitory activity, } \\
\text { laxative, carminative, } \\
\text { antihysteric; treatment of } \\
\text { lumbago, diabetes, rheumatism, } \\
\text { and backache }\end{array}$ & Tehran, & {$[46-49]$} \\
\hline $\begin{array}{l}\text { Ferula flabelliloba } \\
\text { Rech. f. \& Aell. }\end{array}$ & Koma-e Binaloodi & aerial parts, root & $\begin{array}{l}\text { expectorant, aphrodisiac, } \\
\text { sedative, antiseptic, } \\
\text { carminative, antibacterial, } \\
\text { laxative, analgesic, } \\
\text { anthelmintic, diuretic }\end{array}$ & Khorasan & {$[46]$} \\
\hline $\begin{array}{c}\text { Ferulago } \\
\text { carduchorum Boiss } \\
\text { and Hausskn. }\end{array}$ & $\begin{array}{l}\text { No known } \\
\text { common name }\end{array}$ & root & antibacterial activity & Illam, Kerman & {$[50,51]$} \\
\hline $\begin{array}{l}\text { Ferulago contracta } \\
\text { Boiss. \& Hausskn. }\end{array}$ & Chavil-e Khoshei & aerial parts & $\begin{array}{l}\text { sedative, tonic, digestive, } \\
\text { aphrodisiac, and in the } \\
\text { treatment of intestinal worms } \\
\text { and hemorrhoids }\end{array}$ & Yazd & {$[52]$} \\
\hline $\begin{array}{l}\text { Heracleum anisactis } \\
\text { Boiss. }\end{array}$ & Golpar Damavandi & $\begin{array}{l}\text { root, stem, leaf, } \\
\text { and fruit }\end{array}$ & $\begin{array}{l}\text { antiseptic, carminative, } \\
\text { digestive, and analgesic }\end{array}$ & Ardabil & {$[53,54]$} \\
\hline $\begin{array}{c}\text { Heracleum } \\
\text { gorganicum Rech. F. }\end{array}$ & Golpar Gorgani & $\begin{array}{l}\text { root, stem, leaf, } \\
\text { and fruit }\end{array}$ & $\begin{array}{c}\text { carminative, antiseptic, } \\
\text { digestive, epilepsy, } \\
\text { and analgesic }\end{array}$ & Golestan & {$[53,55]$} \\
\hline
\end{tabular}


Table 1. Cont.

\begin{tabular}{|c|c|c|c|c|c|}
\hline Scientific Name & $\begin{array}{l}\text { Common Name } \\
\text { (Vernacular Name) }\end{array}$ & Parts Used & $\begin{array}{l}\text { Therapeutic Effects \& Ethno } \\
\text { Pharmacological Properties } \\
\text { (The Number Inside } \\
\text { Paranthesis of Families Refers } \\
\text { to the Number of } \\
\text { Endemic Species) }\end{array}$ & Province(s) & Ref. \\
\hline $\begin{array}{l}\text { Heracleum rechingeri } \\
\text { Manden. }\end{array}$ & Golpar Asalemi & $\begin{array}{l}\text { root, stem, leaf, } \\
\text { and fruit }\end{array}$ & $\begin{array}{l}\text { carminative, antiseptic, } \\
\text { anthelminthic, diuretic, } \\
\text { digestive, and analgesic }\end{array}$ & Mazandaran & {$[53,56]$} \\
\hline $\begin{array}{l}\text { Kelussia } \\
\text { odoratissima } \\
\text { Mozaff. }\end{array}$ & $\begin{array}{c}\text { Keluss or Karafs-e- } \\
\text { Bakhtiari }\end{array}$ & $\begin{array}{l}\text { leaf, stem, seed, } \\
\text { and root }\end{array}$ & $\begin{array}{l}\text { anti-inflammatory, anti-viral, } \\
\text { anti-diabetic, anti-cancer, } \\
\text { anti-stress, antioxidant, } \\
\text { antihyperlipidemic, ulcerative } \\
\text { colitis, sedative, antibacterial, } \\
\text { pulmonary hypertension, and } \\
\text { anti-tumor }\end{array}$ & $\begin{array}{l}\text { Chaharmahal va } \\
\text { Bakhtiari, Esfahan }\end{array}$ & {$[57,58]$} \\
\hline $\begin{array}{c}\text { Pimpinella } \\
\text { deverroids Boiss. }\end{array}$ & $\begin{array}{l}\text { Jafari kohi or } \\
\text { Anison }\end{array}$ & fruit & antioxidant & $\begin{array}{l}\text { Kermanshah, } \\
\text { Hamadan, Lorestan, } \\
\text { Esfahan, Yazd, Fars, } \\
\text { Chaharmahal } \\
\text { va Bakhtiari }\end{array}$ & [59] \\
\hline $\begin{array}{c}\text { Prangos } \\
\text { cheilanthifolia Boiss. }\end{array}$ & Joshire Azarbaijani & aerial parts & $\begin{array}{c}\text { emollient, carminative, } \\
\text { antifungal, antioxidant, } \\
\text { antibacterial, anti-HIV, tonic, } \\
\text { antiflatulent, anthelmintic }\end{array}$ & $\begin{array}{c}\text { East and West } \\
\text { Azarbaijan, Esfahan, } \\
\text { Yazd, Kerman, Tehran }\end{array}$ & {$[60]$} \\
\hline \multicolumn{6}{|c|}{ Asteraceae (7) } \\
\hline $\begin{array}{l}\text { Achillea aucheri } \\
\text { Boiss. }\end{array}$ & $\begin{array}{l}\text { Boomadaran } \\
\text { Damavandi }\end{array}$ & $\begin{array}{l}\text { aerial parts with } \\
\text { flowering tops }\end{array}$ & $\begin{array}{l}\text { antispasmodic, } \\
\text { anti-inflammatory, diuretic, and } \\
\text { diaphoretic, treatment of } \\
\text { hemorrhage, pneumonia, } \\
\text { rheumatic pain, and wounds }\end{array}$ & $\begin{array}{c}\text { East and West } \\
\text { Azarbaijan, Tehran }\end{array}$ & {$[61-63]$} \\
\hline $\begin{array}{c}\text { Achillea biebersteinii } \\
\text { Afan. }\end{array}$ & Bumadaran-e Zard & $\begin{array}{l}\text { aerial parts with } \\
\text { flowering tops }\end{array}$ & $\begin{array}{l}\text { hypoglycemic, nerve tonic, } \\
\text { anti-hemorrhoid, antidiarrhea, } \\
\text { antacid, carminative, appetizer, } \\
\text { anthelmintic and antibacterial }\end{array}$ & $\begin{array}{c}\text { Hamadan, } \\
\text { East-Azarbaijan }\end{array}$ & {$[63-65]$} \\
\hline $\begin{array}{c}\text { Achillea eriophora de } \\
\text { Candolle. }\end{array}$ & $\begin{array}{l}\text { Bumadaran-e } \\
\text { Shirazi }\end{array}$ & $\begin{array}{l}\text { aerial parts with } \\
\text { flowering tops }\end{array}$ & $\begin{array}{l}\text { feverish conditions, common } \\
\text { cold, digestive complaints, slow } \\
\text { healing wounds, and } \\
\text { skin inflammations }\end{array}$ & $\begin{array}{c}\text { Sistan va Baluchestan, } \\
\text { Fars, Hormozgan, } \\
\text { Khuzestan, Kerman }\end{array}$ & {$[63,64,66,67]$} \\
\hline $\begin{array}{l}\text { Achillea kellalensis } \\
\text { Boiss. \& Hausskn. }\end{array}$ & $\begin{array}{l}\text { Bumadaran-e } \\
\text { Bakhtiari } \\
\text { Golberrenjas } \\
\text { Bumadaran-e- } \\
\text { Sabzekoh }\end{array}$ & $\begin{array}{l}\text { aerial parts with } \\
\text { flowering tops }\end{array}$ & $\begin{array}{l}\text { remedy for edema, burns, } \\
\text { wounds, carminative, } \\
\text { indigestion, skin infection, } \\
\text { gastric ulcer, anti-bacterial, } \\
\text { hemorrhage, dysmenorrhoea, } \\
\text { enema, and diarrhea }\end{array}$ & $\begin{array}{l}\text { Lorestan, Esfahan, } \\
\text { Chaharmahal va } \\
\text { Bakhtiari, Fars }\end{array}$ & {$[63]$} \\
\hline $\begin{array}{l}\text { Achillea oxyodonta } \\
\text { Boiss. }\end{array}$ & $\begin{array}{l}\text { Bumadaran-e } \\
\text { Shemirani }\end{array}$ & $\begin{array}{l}\text { aerial parts with } \\
\text { flowering tops }\end{array}$ & $\begin{array}{l}\text { spasmolytic, choleretic, } \\
\text { treatment of wounds, and } \\
\text { anti-inflammatory activities }\end{array}$ & $\begin{array}{c}\text { Tehran, Hamadan, } \\
\text { Esfahan }\end{array}$ & {$[63,68]$} \\
\hline $\begin{array}{c}\text { Achillea talagonica } \\
\text { Boiss. }\end{array}$ & $\begin{array}{l}\text { Bumadaran-e } \\
\text { Taleghani }\end{array}$ & $\begin{array}{l}\text { aerial parts with } \\
\text { flowering tops }\end{array}$ & $\begin{array}{l}\text { fever, asthma, skin } \\
\text { inflammation, jaundice, and } \\
\text { liver ailments. }\end{array}$ & $\begin{array}{l}\text { Chaharmahal va } \\
\text { Bakhtiari, Tehran, } \\
\text { Khuzestan, East } \\
\text { and West } \\
\text { Azarbaijan, Kurdestan, } \\
\text { Lorestan, Esfahan }\end{array}$ & {$[63,69,70]$} \\
\hline $\begin{array}{l}\text { Postia puberula } \\
\text { Boiss. \& Blanche }\end{array}$ & $\begin{array}{l}\text { No known } \\
\text { common name }\end{array}$ & aerial parts & antioxidant activity & Lorestan & {$[71]$} \\
\hline \multicolumn{6}{|c|}{ Boraginaceae (2) } \\
\hline $\begin{array}{c}\text { Echium amoenum } \\
\text { Fisch. \& C.A. Mey. }\end{array}$ & $\begin{array}{l}\text { Gol-e-Gavzaban } \\
\text { Irani }\end{array}$ & petal & $\begin{array}{l}\text { tonic, tranquilizer, diaphoretic, } \\
\text { cough suppressant, and a } \\
\text { remedy for sore throat } \\
\text { and pneumonia, }\end{array}$ & $\begin{array}{c}\text { Golestan, East and west } \\
\text { Azarbaijan, } \\
\text { Mazandaran, } \\
\text { Gilan, Hamadan }\end{array}$ & {$[72,73]$} \\
\hline
\end{tabular}


Table 1. Cont.

\begin{tabular}{|c|c|c|c|c|c|}
\hline Scientific Name & $\begin{array}{c}\text { Common Name } \\
\text { (Vernacular Name) }\end{array}$ & Parts Used & $\begin{array}{l}\text { Therapeutic Effects \& Ethno } \\
\text { Pharmacological Properties } \\
\text { (The Number Inside } \\
\text { Paranthesis of Families Refers } \\
\text { to the Number of } \\
\text { Endemic Species) }\end{array}$ & Province(s) & Ref. \\
\hline $\begin{array}{l}\text { Echium } \\
\text { khuzistanicum } \\
\text { Mozaffarian. }\end{array}$ & $\begin{array}{l}\text { Gol-e-Gavzaban } \\
\text { Khuzestani }\end{array}$ & petal & $\begin{array}{c}\text { anti-allergic, antibacterial, } \\
\text { antiviral, antifungal, } \\
\text { antioxidant, anti-inflammatory, } \\
\text { and wound healing }\end{array}$ & Khuzestan & {$[74]$} \\
\hline \multicolumn{6}{|c|}{ Fabaceae (8) } \\
\hline $\begin{array}{c}\text { Astragalus } \\
\text { adscendens } \\
\text { Boiss. \& Hausskn. }\end{array}$ & $\begin{array}{l}\text { Gaz-e Khansar } \\
\text { Gavan-e Gaz- } \\
\text { Angabin }\end{array}$ & $\begin{array}{l}\text { stem, leaf, flower, } \\
\text { root, manna }\end{array}$ & $\begin{array}{c}\text { antioxidant, laxative, } \\
\text { antispasmodic, antiheadache, } \\
\text { antidiabetic, febrifuge, } \\
\text { and digestive }\end{array}$ & $\begin{array}{c}\text { Esfahan, Chaharmahal } \\
\text { va Bakhtiari, Lorestan } \\
\text { and Khuzestan }\end{array}$ & {$[75,76]$} \\
\hline $\begin{array}{c}\text { Astragalus } \\
\text { fasciculifolius Boiss. }\end{array}$ & $\begin{array}{l}\text { Anzrot } \\
\text { Gonjed }\end{array}$ & $\begin{array}{l}\text { leaf, flower, root, } \\
\text { stem, seed }\end{array}$ & $\begin{array}{l}\text { tightening the roots of teeth, } \\
\text { cough, nutritious, kidney, } \\
\text { stomach ache, chest infection, } \\
\text { toothache treating heart disease } \\
\text { and cancer }\end{array}$ & Sistan va Baluchestan & {$[76]$} \\
\hline $\begin{array}{c}\text { Astragalus } \\
\text { gossypinus Fisch. }\end{array}$ & Gavan-e Panbei & gum & $\begin{array}{l}\text { cough, anti-fungal, skin } \\
\text { diseases, hair gel }\end{array}$ & Kermanshah & {$[76]$} \\
\hline $\begin{array}{l}\text { Astragalus hamosus } \\
\text { Linnaeus. }\end{array}$ & $\begin{array}{l}\text { Iklil-ul-Malik } \\
\text { Nakhonak }\end{array}$ & pod & $\begin{array}{l}\text { headache, vertigo, strokes and } \\
\text { dementia gastrointestinal upset, } \\
\text { inflammations, respiratory } \\
\text { discomfort, and } \\
\text { urinary complications }\end{array}$ & $\begin{array}{l}\text { Esfahan, Fars } \\
\text { and Bushehr }\end{array}$ & [77] \\
\hline $\begin{array}{l}\text { Astragalus fischeri } \\
\text { Buhse ex Fisch. }\end{array}$ & Shoun korouchok & $\begin{array}{l}\text { aerial parts, } \\
\text { seed, root }\end{array}$ & $\begin{array}{l}\text { toothache, backache, bone ache, } \\
\text { kidney ache, bone fracture, } \\
\text { diabetes, and to induce abortion }\end{array}$ & $\begin{array}{l}\text { Esfahan, Fars } \\
\text { and Bushehr }\end{array}$ & {$[76]$} \\
\hline $\begin{array}{c}\text { Astragalus } \\
\text { microcephalus Willd. }\end{array}$ & Kalelak- & stem, root & asthma, strengthen hair & Tehran, Mazandaran & {$[76]$} \\
\hline $\begin{array}{c}\text { Astragalus } \\
\text { Chrysostachys Boiss. }\end{array}$ & Gavan & root & antioxidant and antibacterial & East Azarbaijan & {$[78]$} \\
\hline $\begin{array}{l}\text { Astragalus } \\
\text { Podolobus } \\
\text { Boiss. \& Hohen. }\end{array}$ & Katek & $\begin{array}{l}\text { aerial parts, leaf, } \\
\text { flower, seed }\end{array}$ & anemia & Hormozgan & {$[79]$} \\
\hline \multicolumn{6}{|c|}{ Hypericaceae (2) } \\
\hline $\begin{array}{c}\text { Hypericum } \\
\text { asperulum } \\
\text { Jaub. \& Spach. }\end{array}$ & $\begin{array}{l}\text { Gol-e Raei } \\
\text { Lorestani }\end{array}$ & $\begin{array}{l}\text { flowering aerial } \\
\text { parts }\end{array}$ & $\begin{array}{l}\text { anti-depression, sedative, } \\
\text { strengthens the nervous system } \\
\text { and antioxidant }\end{array}$ & Kurdestan & {$[80]$} \\
\hline $\begin{array}{c}\text { Hypericum } \\
\text { dogonbadanicum } \\
\text { Assadi. }\end{array}$ & Hofariqun & $\begin{array}{l}\text { flowering aerial } \\
\text { parts }\end{array}$ & $\begin{array}{l}\text { anti-depression, sedative, } \\
\text { strengthens the nervous system } \\
\text { and antioxidant }\end{array}$ & $\begin{array}{l}\text { Kuhkiluye va } \\
\text { Boyer-Ahmad }\end{array}$ & {$[80,81]$} \\
\hline \multicolumn{6}{|c|}{ Lamiaceae (55) } \\
\hline $\begin{array}{c}\text { Ajuga chamaecistus } \\
\text { Ging. }\end{array}$ & Labdisi & aerial parts & $\begin{array}{c}\text { hypoglycemic, } \\
\text { anti-inflammatory, analgesic, } \\
\text { anti-arthritis, antipyretic, } \\
\text { hepatoprotective, antibacterial, } \\
\text { antifungal, antioxidant, } \\
\text { cardiotonic, and antimalarial }\end{array}$ & $\begin{array}{c}\text { Tehran, Semnan, East } \\
\text { and West Azarbaijan, } \\
\text { Hamadan, } \\
\text { Kermanshah, } \\
\text { Arak, Esfahan }\end{array}$ & {$[82,83]$} \\
\hline $\begin{array}{l}\text { Dracocephalum } \\
\text { kotschyi Boiss. }\end{array}$ & $\begin{array}{l}\text { Badranjboye } \\
\text { Denayi }\end{array}$ & aerial parts & $\begin{array}{l}\text { antioxidant, antibacterial, } \\
\text { anticancerous, antinociceptive, } \\
\text { antihyperlipidemic, } \\
\text { antispasmodic, cytotoxic, and } \\
\text { immunomodulatory effects }\end{array}$ & $\begin{array}{c}\text { Esfahan, Yasuj, } \\
\text { Mazandaran, Tabriz, } \\
\text { Golestan, Hamadan, } \\
\text { Fars, Semnan, Tehran }\end{array}$ & [84-86] \\
\hline $\begin{array}{l}\text { Dracocephalum } \\
\text { polychaetum } \\
\text { Linnaeus. }\end{array}$ & $\begin{array}{l}\text { Badranjboye } \\
\text { Kermani }\end{array}$ & aerial parts & $\begin{array}{l}\text { anti-depression, anticancer, } \\
\text { antimicrobial, and } \\
\text { vasodilative effects }\end{array}$ & Kerman & {$[87,88]$} \\
\hline
\end{tabular}


Table 1. Cont.

\begin{tabular}{|c|c|c|c|c|c|}
\hline Scientific Name & $\begin{array}{l}\text { Common Name } \\
\text { (Vernacular Name) }\end{array}$ & Parts Used & $\begin{array}{l}\text { Therapeutic Effects \& Ethno } \\
\text { Pharmacological Properties } \\
\text { (The Number Inside } \\
\text { Paranthesis of Families Refers } \\
\text { to the Number of } \\
\text { Endemic Species) }\end{array}$ & Province(s) & Ref. \\
\hline $\begin{array}{l}\text { Dracocephalum } \\
\text { surmandinum } \\
\text { Rech. f. }\end{array}$ & $\begin{array}{l}\text { Badranjboye } \\
\text { Sormandi }\end{array}$ & aerial parts & $\begin{array}{c}\text { tonic, and } \\
\text { gastrointestinal disorders }\end{array}$ & $\begin{array}{c}\text { Esfahan, Chaharmahal } \\
\text { va Bakhtiari }\end{array}$ & [89] \\
\hline $\begin{array}{l}\text { Hymenocrater } \\
\text { yazdianus Rech.f. }\end{array}$ & Gol-e Arvane Yazdi & leaves & $\begin{array}{l}\text { antimicrobial, antiparasitic, } \\
\text { antioxidant, anticancer and } \\
\text { antidiabetic activities }\end{array}$ & Yazd & {$[90]$} \\
\hline $\begin{array}{l}\text { Mentha } \\
\text { mozaffarianii } \\
\text { Jamzad. }\end{array}$ & Pooneh-Kooh & $\begin{array}{l}\text { aerial parts, leaves, } \\
\text { and seeds }\end{array}$ & $\begin{array}{l}\text { stomachache, cramps, chest } \\
\text { pain, bronchitis, and colds }\end{array}$ & Hormozgan, Fars & {$[91,92]$} \\
\hline $\begin{array}{l}\text { Nepeta binalodensis } \\
\text { Jamzad. }\end{array}$ & Binaludi Pune-sa & aerial parts & $\begin{array}{l}\text { headache, migraine, digestive, } \\
\text { rheumatism, respiratory } \\
\text { disorders, asthma, common } \\
\text { cold, colic and } \\
\text { cardio-vascular disorders }\end{array}$ & Khorasan & {$[25,93,94]$} \\
\hline $\begin{array}{l}\text { Nepeta cephalotes } \\
\text { Boiss. }\end{array}$ & Kopei Pune-sa & aerial parts & $\begin{array}{c}\text { diuretic, diaphoretic, } \\
\text { antitussive, antispasmodic, } \\
\text { anti-asthmatic, febrifuge, } \\
\text { emmenagogue and } \\
\text { sedative agents }\end{array}$ & Tehran & [25] \\
\hline $\begin{array}{l}\text { Nepeta crassifolia } \\
\text { Boiss. \& Buhse }\end{array}$ & Alborzi Pune-sa & aerial parts & $\begin{array}{l}\text { cardiovascular complaints such } \\
\text { as angina pectoris, cardiac } \\
\text { thrombosis, tachycardia, and } \\
\text { weakness of the heart }\end{array}$ & $\begin{array}{l}\text { Ardabil, East } \\
\text { Azarbaijan }\end{array}$ & {$[25,26,95,96]$} \\
\hline Nepeta crispa Willd. & Mavaj Pune-sa & aerial parts & $\begin{array}{c}\text { sedative, relaxant, carminative, } \\
\text { tonic for respiratory and } \\
\text { nervous disorders }\end{array}$ & $\begin{array}{c}\text { Hamadan, } \\
\text { Kermanshah, } \\
\text { Chaharmahal va } \\
\text { Bakhtiari, Tehran }\end{array}$ & {$[25,97]$} \\
\hline $\begin{array}{l}\text { Nepeta denudata } \\
\text { Benth. }\end{array}$ & Oryan Pune-sa & aerial parts & $\begin{array}{c}\text { diuretic, diaphoretic, } \\
\text { antitussive, antispasmodic, } \\
\text { anti-asthmatic, febrifuge, } \\
\text { emmenagogue and } \\
\text { sedative agents }\end{array}$ & Tehran & [25] \\
\hline $\begin{array}{l}\text { Nepeta Depauperata } \\
\text { Benth. }\end{array}$ & $\begin{array}{l}\text { Sabzposhani } \\
\text { Pune-sa }\end{array}$ & aerial parts & $\begin{array}{c}\text { diuretic, diaphoretic, } \\
\text { antitussive, antispasmodic, } \\
\text { anti-asthmatic, febrifuge, } \\
\text { emmenagogue and } \\
\text { sedative agents }\end{array}$ & Kerman & {$[25,98]$} \\
\hline $\begin{array}{l}\text { Nepeta } \\
\text { dschuparensis } \\
\text { Bornm. }\end{array}$ & Jupari Pune-sa & aerial parts & $\begin{array}{c}\text { diuretic, diaphoretic, } \\
\text { antitussive, antispasmodic, } \\
\text { anti-asthmatic, febrifuge, } \\
\text { emmenagogue and } \\
\text { sedative agents }\end{array}$ & Fars, Kerman & {$[25,99]$} \\
\hline $\begin{array}{l}\text { Nepeta elymaitica } \\
\text { Bornm. }\end{array}$ & Ilami Pune-sa & aerial parts & $\begin{array}{l}\text { diaphoretic, antitussive, } \\
\text { antispasmodic, anti-asthmatic, } \\
\text { febrifuge, emmenagogue, } \\
\text { sedative, and diuretic }\end{array}$ & Esfahan & {$[25]$} \\
\hline $\begin{array}{c}\text { Nepeta schiraziana } \\
\text { Boiss. }\end{array}$ & Shirazi Pune-sa & aerial parts & $\begin{array}{l}\text { antitussive, antispasmodic, } \\
\text { anti-asthmatic, febrifuge, } \\
\text { emmenagogue, sedative, } \\
\text { diuretic, and diaphoretic }\end{array}$ & $\begin{array}{l}\text { Lorestan, Esfahan, } \\
\text { Chaharmahal va } \\
\text { Bakhtiari, Fars, } \\
\text { Khorasan, Semnan }\end{array}$ & {$[25]$} \\
\hline $\begin{array}{c}\text { Nepeta glomerulosa } \\
\text { Boiss }\end{array}$ & Anboh Pune-sa & aerial parts & $\begin{array}{l}\text { antispasmodic, anti-asthmatic, } \\
\text { febrifuge, emmenagogue, } \\
\text { sedative, diuretic, diaphoretic, } \\
\text { and antitussive }\end{array}$ & $\begin{array}{c}\text { Mazandaran, Esfahan, } \\
\text { Fars, Kerman, } \\
\text { Khorasan, Tehran }\end{array}$ & {$[25,100]$} \\
\hline
\end{tabular}


Table 1. Cont.

\begin{tabular}{|c|c|c|c|c|c|}
\hline Scientific Name & $\begin{array}{l}\text { Common Name } \\
\text { (Vernacular Name) }\end{array}$ & Parts Used & $\begin{array}{l}\text { Therapeutic Effects \& Ethno } \\
\text { Pharmacological Properties } \\
\text { (The Number Inside } \\
\text { Paranthesis of Families Refers } \\
\text { to the Number of } \\
\text { Endemic Species) }\end{array}$ & Province(s) & Ref. \\
\hline $\begin{array}{c}\text { Nepeta heliotropifolia } \\
\text { Lam. }\end{array}$ & $\begin{array}{l}\text { Aftab-parasti } \\
\text { Pune-sa }\end{array}$ & aerial parts & $\begin{array}{l}\text { anti-asthmatic, febrifuge, } \\
\text { emmenagogue, sedative, } \\
\text { diuretic, diaphoretic, } \\
\text { antitussive, and antispasmodic }\end{array}$ & Markazi, Qazvin & {$[25,101]$} \\
\hline $\begin{array}{l}\text { Nepeta involucrate } \\
\text { Bornm. }\end{array}$ & $\begin{array}{l}\text { Gariban dar } \\
\text { Pune-sa }\end{array}$ & aerial parts & $\begin{array}{l}\text { febrifuge, emmenagogue, } \\
\text { sedative, diuretic, diaphoretic, } \\
\text { antitussive, antispasmodic, } \\
\text { and anti-asthmatic }\end{array}$ & - & [25] \\
\hline $\begin{array}{c}\text { Nepeta ispahanica } \\
\text { Boiss. }\end{array}$ & Esfahani Pune-sa & aerial parts & $\begin{array}{c}\text { emmenagogue, sedative, } \\
\text { diuretic, diaphoretic, } \\
\text { antitussive, antispasmodic, } \\
\text { anti-asthmatic, and febrifuge }\end{array}$ & Esfahan & {$[25,94]$} \\
\hline $\begin{array}{l}\text { Nepeta kotschyi } \\
\text { Boiss. }\end{array}$ & Kohe Dalv Pune-sa & aerial parts & $\begin{array}{l}\text { sedative, diuretic, diaphoretic, } \\
\text { antitussive, antispasmodic, } \\
\text { anti-asthmatic, febrifuge, } \\
\text { and emmenagogue }\end{array}$ & - & [25] \\
\hline $\begin{array}{l}\text { Nepeta mentoides } \\
\text { Boiss. \& Buhse. }\end{array}$ & Sabalani Pune-sa & aerial parts & $\begin{array}{l}\text { antispasmodic, anti-asthmatic, } \\
\text { febrifuge, emmenagogue, } \\
\text { sedative, diuretic, diaphoretic, } \\
\text { and antitussive }\end{array}$ & $\begin{array}{c}\text { East and West } \\
\text { Azarbaijan }\end{array}$ & {$[25,102]$} \\
\hline Nepeta persica Boiss. & Irani Pune-sa & aerial parts & $\begin{array}{l}\text { febrifuge, emmenagogue, } \\
\text { sedative, diuretic, diaphoretic, } \\
\text { antitussive, antispasmodic, } \\
\text { and anti-asthmatic }\end{array}$ & Ardabil, Esfahan & {$[25,96,103]$} \\
\hline $\begin{array}{l}\text { Nepeta rivularis } \\
\text { Bornm. }\end{array}$ & Juybari Pune-sa & aerial parts & $\begin{array}{l}\text { emmenagogue, sedative, } \\
\text { diuretic, diaphoretic, } \\
\text { antitussive, antispasmodic, } \\
\text { anti-asthmatic, and febrifuge }\end{array}$ & Kerman & {$[25]$} \\
\hline $\begin{array}{l}\text { Nepeta sintenisii } \\
\text { Bornm. }\end{array}$ & Torkamani Pune-sa & aerial parts & $\begin{array}{c}\text { diuretic, diaphoretic, } \\
\text { antitussive, antispasmodic, } \\
\text { anti-asthmatic, febrifuge, } \\
\text { emmenagogue and } \\
\text { sedative agents }\end{array}$ & Mazandaran & {$[25,96,104]$} \\
\hline $\begin{array}{l}\text { Otostegia persica } \\
\text { Boiss. }\end{array}$ & $\begin{array}{c}\text { Golder, } \\
\text { Gol-e-kharu }\end{array}$ & $\begin{array}{l}\text { top flowering } \\
\text { aerial parts }\end{array}$ & $\begin{array}{l}\text { antispasmodic, antihistaminic, } \\
\text { antimalarial, anti-arthritis, } \\
\text { diabetes, arthritis, gastric } \\
\text { discomfort, headache, } \\
\text { rheumatism, sedative activities, } \\
\text { regulating blood pressure, } \\
\text { and hyperlipidemia. }\end{array}$ & $\begin{array}{l}\text { Fars, Kerman, Sistan } \\
\text { va Baluchestan }\end{array}$ & [105-108] \\
\hline $\begin{array}{c}\text { Satureja avromanica } \\
\text { Maroofi. }\end{array}$ & Marzeh Oramani & aerial parts & $\begin{array}{c}\text { antimicrobial, antioxidant, } \\
\text { antispasmodic, } \\
\text { and anti-diarrheal }\end{array}$ & Kurdestan & {$[106,108]$} \\
\hline $\begin{array}{l}\text { Satureja Edmondi } \\
\text { Briquet. }\end{array}$ & Marzeh Edmondi & aerial parts & $\begin{array}{l}\text { antimicrobial, antioxidant, } \\
\text { antiviral activity (against HIV), } \\
\text { and improvement of fertility }\end{array}$ & $\begin{array}{c}\text { Kermanshah, Lorestan, } \\
\text { Chaharmahal } \\
\text { va Bakhtiari }\end{array}$ & {$[109,110]$} \\
\hline $\begin{array}{c}\text { Satureja atropatana } \\
\text { Bunge. }\end{array}$ & $\begin{array}{c}\text { Marzeh } \\
\text { Azarbaijani }\end{array}$ & aerial parts & $\begin{array}{c}\text { gastroenteritis, } \\
\text { upperrespiratory tract } \\
\text { infections, urinary tract } \\
\text { infections, diarrhea, and } \\
\text { wound healing }\end{array}$ & $\begin{array}{c}\text { East and west } \\
\text { Azarbaijan }\end{array}$ & {$[109,110]$} \\
\hline $\begin{array}{c}\text { Satureja bachtiarica } \\
\text { Bunge }\end{array}$ & Marzeh Bakhtiari & aerial parts & $\begin{array}{l}\text { antimicrobial, antioxidant, } \\
\text { antispasmodic, anti-diarrheal, } \\
\text { and antitumor activities }\end{array}$ & $\begin{array}{c}\text { Kurdestan, } \\
\text { Kermanshah, Lorestan, } \\
\text { Chaharmahal va } \\
\text { Bakhtiari, Fars }\end{array}$ & [109-112] \\
\hline
\end{tabular}


Table 1. Cont.

\begin{tabular}{|c|c|c|c|c|c|}
\hline Scientific Name & $\begin{array}{l}\text { Common Name } \\
\text { (Vernacular Name) }\end{array}$ & Parts Used & $\begin{array}{l}\text { Therapeutic Effects \& Ethno } \\
\text { Pharmacological Properties } \\
\text { (The Number Inside } \\
\text { Paranthesis of Families Refers } \\
\text { to the Number of } \\
\text { Endemic Species) }\end{array}$ & Province(s) & Ref. \\
\hline $\begin{array}{l}\text { Satureja intermedia } \\
\text { C. A. Mey. }\end{array}$ & Marzeh Taleshi & aerial parts & $\begin{array}{l}\text { upper respiratory tract } \\
\text { infections, urinary tract } \\
\text { infections, diarrhea, wounds, } \\
\text { and gastroenteritis }\end{array}$ & Gilan, Ardabil & {$[109,110]$} \\
\hline $\begin{array}{l}\text { Satureja isophylla } \\
\text { Rech. f. }\end{array}$ & Marzeh Jorbarg & aerial parts & $\begin{array}{l}\text { urinary tract infections, } \\
\text { diarrhea, wounds, } \\
\text { gastroenteritis, and upper } \\
\text { respiratory tract infections }\end{array}$ & Mazandaran & {$[109,110]$} \\
\hline $\begin{array}{l}\text { Satureja kallarica } \\
\text { Jamzad. }\end{array}$ & Marzeh Kellari & aerial parts & $\begin{array}{l}\text { diarrhea, wounds, } \\
\text { gastroenteritis, upper } \\
\text { respiratory tract infections, and } \\
\text { urinary tract infections }\end{array}$ & $\begin{array}{c}\text { Chaharmahal va } \\
\text { Bakhtiari }\end{array}$ & [106] \\
\hline $\begin{array}{l}\text { Satureja } \\
\text { Kermanshahensis } \\
\text { Jamzad. }\end{array}$ & $\begin{array}{c}\text { Marzeh } \\
\text { Kermanshahi }\end{array}$ & aerial parts & $\begin{array}{l}\text { wounds, gastroenteritis, upper } \\
\text { respiratory tract infections, } \\
\text { urinary tract infections, } \\
\text { and diarrhea }\end{array}$ & Kermanshah & [106] \\
\hline $\begin{array}{c}\text { Satureja khuzistanica } \\
\text { Jamzad. }\end{array}$ & Marzeh Khuzistani & aerial parts & $\begin{array}{c}\text { antifungal, antibacterial, } \\
\text { antinociceptive, antioxidant, } \\
\text { antidiabetic, } \\
\text { antihyperlipidemic, } \\
\text { anti-inflammatory, and } \\
\text { triglyceride-lowering activities }\end{array}$ & Khuzestan & [113] \\
\hline $\begin{array}{c}\text { Satureja rechingeri } \\
\text { Jamzad. }\end{array}$ & Marzeh rechingeri & aerial parts & $\begin{array}{l}\text { antioxidant, antidiabetic, } \\
\text { antihyperlipidemic, } \\
\text { anti-inflammatory, } \\
\text { antifungal, antibacterial, } \\
\text { antinociceptive, and } \\
\text { triglyceride-lowering activities }\end{array}$ & Ilam & {$[103,106,107]$} \\
\hline $\begin{array}{l}\text { Satureja sahandica } \\
\text { Bormn. }\end{array}$ & Marzeh Sahandi & aerial parts & $\begin{array}{l}\text { gastroenteritis, upper } \\
\text { respiratory tract infections, } \\
\text { urinary tract infections, } \\
\text { diarrhea, and wounds }\end{array}$ & $\begin{array}{c}\text { East and } \\
\text { West Azarbaijan, } \\
\text { Kurdestan, Zanjan }\end{array}$ & [109] \\
\hline $\begin{array}{l}\text { Stachys acerosa } \\
\text { Boiss. }\end{array}$ & $\begin{array}{l}\text { Sonboleh Kohsari } \\
\text { Sonboleh } \\
\text { Kharaloud }\end{array}$ & aerial parts & $\begin{array}{c}\text { antispasmodic, diuretic, } \\
\text { asthmatic, rheumatic } \\
\text { antibacterial, and antioxidant }\end{array}$ & $\begin{array}{l}\text { Kerman, Hamadan, } \\
\text { Lorestan, Arak, } \\
\text { Esfahan, Kohkilouyeh } \\
\text { va Boyer-Ahmad, } \\
\text { Chaharmahal va } \\
\text { Bakhtiari, Fars }\end{array}$ & {$[114,115]$} \\
\hline $\begin{array}{l}\text { Stachys asterocalyx } \\
\text { Rech. } \mathrm{f} .\end{array}$ & Sonboleh Shirazi & aerial parts & $\begin{array}{l}\text { genital tumors, sclerosis of the } \\
\text { spleen, inflammatory tumors, } \\
\text { cough, and ulcers }\end{array}$ & Fars & {$[116]$} \\
\hline $\begin{array}{c}\text { Stachys benthamiana } \\
\text { Boiss. }\end{array}$ & $\begin{array}{c}\text { Sonboleh Sakhreh } \\
\text { Zei }\end{array}$ & aerial parts & $\begin{array}{c}\text { antibacterial, antifungal, } \\
\text { antioxidant, anxiolytic, } \\
\text { anti-inflammatory, hypotensive, } \\
\text { and anti-nephritic activities }\end{array}$ & $\begin{array}{c}\text { Fars, Chaharmahal va } \\
\text { Bakhtiari, Esfahan }\end{array}$ & {$[114,115]$} \\
\hline $\begin{array}{l}\text { Stachys laxa } \\
\text { Boiss. \& Buhse. }\end{array}$ & $\begin{array}{l}\text { Sonboleh } \\
\text { Damavandi }\end{array}$ & aerial parts & $\begin{array}{l}\text { anticancer, antibacterial, } \\
\text { antioxidant, anti-inflammatory, } \\
\text { anti-nephritic, anti-anxiety }\end{array}$ & $\begin{array}{l}\text { Golestan, Mazandaran, } \\
\text { Semnan, Tehran }\end{array}$ & {$[117,118]$} \\
\hline $\begin{array}{c}\text { Stachys obtusicrena } \\
\text { Boiss. }\end{array}$ & Sonboleh Kongerei & aerial parts & $\begin{array}{l}\text { genital tumors, sclerosis of the } \\
\text { spleen, inflammatory tumors, } \\
\text { cough, ulcers, and } \\
\text { infected wounds }\end{array}$ & $\begin{array}{c}\text { East and West } \\
\text { Azarbaijan, Yazd, Gilan }\end{array}$ & [116] \\
\hline
\end{tabular}


Table 1. Cont.

\begin{tabular}{|c|c|c|c|c|c|}
\hline Scientific Name & $\begin{array}{c}\text { Common Name } \\
\text { (Vernacular Name) }\end{array}$ & Parts Used & $\begin{array}{l}\text { Therapeutic Effects \& Ethno } \\
\text { Pharmacological Properties } \\
\text { (The Number Inside } \\
\text { Paranthesis of Families Refers } \\
\text { to the Number of } \\
\text { Endemic Species) }\end{array}$ & Province(s) & Ref. \\
\hline $\begin{array}{l}\text { Stachys pilifera } \\
\text { Benth. }\end{array}$ & Sonboleh Modar & aerial parts & $\begin{array}{l}\text { asthma, rheumatoid arthritis, } \\
\text { antioxidant, antimicrobial, } \\
\text { anti-inflammatory, } \\
\text { and antitumor }\end{array}$ & Yasuj & {$[119,120]$} \\
\hline $\begin{array}{c}\text { Thymus carmanicus } \\
\text { Jalas. }\end{array}$ & Avishan-e-kermani & aerial parts & $\begin{array}{l}\text { antispasmodic, antimycotic, } \\
\text { mammalian age-delaying } \\
\text { properties, bactericides, } \\
\text { antiseptics, antioxidants, and } \\
\text { anthelmintic properties }\end{array}$ & Kerman & {$[112,121]$} \\
\hline $\begin{array}{l}\text { Thymus deanensis } \\
\text { Celak. }\end{array}$ & Avishan-e-denaee & aerial parts & antibacterial & $\begin{array}{l}\text { Hamadan, Azarbaijan, } \\
\text { Chaharmahal va } \\
\text { Bakhtiari, Kurdestan, } \\
\text { Hamadan, } \\
\text { Kermanshah, Esfahan, } \\
\text { Tehran, Fars, Kerman }\end{array}$ & {$[112,121-125]$} \\
\hline $\begin{array}{l}\text { Thymus eriocalyx } \\
\text { (Ronninger.) Jalas. }\end{array}$ & $\begin{array}{l}\text { Avishan-e- } \\
\text { Korkalod }\end{array}$ & aerial parts & gastrointestinal disturbances & Lorestan & {$[124,125]$} \\
\hline $\begin{array}{l}\text { Thymus fallax Fisch. } \\
\text { \& C. A. Mey. }\end{array}$ & Avishan-e-Anatoli & aerial parts & $\begin{array}{c}\text { antibacterial, antifungal, } \\
\text { antiviral, antiparasitic, } \\
\text { spasmolytic, and antioxidant }\end{array}$ & Hamadan, Tehran & {$[126,127]$} \\
\hline $\begin{array}{l}\text { Thymus kotschyanus } \\
\text { Boiss. \& Hohen. }\end{array}$ & Avishan & aerial parts & $\begin{array}{l}\text { gastrointestinal disturbances, } \\
\text { anthelmintic, antioxidant, } \\
\text { strongly antiseptic, } \\
\text { antispasmodic, carminative, } \\
\text { deodorant, diaphoretic, } \\
\text { disinfectant, expectorant, } \\
\text { sedative, and tonic }\end{array}$ & $\begin{array}{l}\text { Mazandaran, Gilan, } \\
\text { East, and West } \\
\text { Azarbaijan, Tehran, } \\
\text { Kurdestan, Yazd }\end{array}$ & $\begin{array}{c}{[121,124,128,} \\
129]\end{array}$ \\
\hline $\begin{array}{l}\text { Thymus persicus } \\
\text { (Ronniger ex } \\
\text { Rech. f.) Jalas. }\end{array}$ & Avishan-e-Irani & aerial parts & $\begin{array}{l}\text { anti-inflammatory, } \\
\text { hepatoprotective, antitumor, } \\
\text { anti-HIV, antimicrobial, } \\
\text { antifungal, anti-ulcer, } \\
\text { gastroprotective, hypoglycemic, } \\
\text { and antihyperlipidemic }\end{array}$ & $\begin{array}{c}\text { East and West } \\
\text { Azarbaijan }\end{array}$ & {$[125,130]$} \\
\hline $\begin{array}{l}\text { Thymus pubescens } \\
\text { Boiss. \& Kotschy } \\
\quad \text { ex Celak. }\end{array}$ & $\begin{array}{l}\text { Avishan-e- } \\
\text { korkaloud }\end{array}$ & aerial parts & $\begin{array}{l}\text { tonic, carminative, digestive, } \\
\text { antispasmodic, } \\
\text { anti-inflammatory, } \\
\text { and expectorant }\end{array}$ & $\begin{array}{c}\text { East and West } \\
\text { Azarbaijan, } \\
\text { Mazandaran, Tehran }\end{array}$ & {$[121]$} \\
\hline $\begin{array}{c}\text { Thymus trautvetteri } \\
\text { Klokov \& } \\
\text { Desj.- Shost. }\end{array}$ & Avishan-e-Taleshi & aerial parts & $\begin{array}{l}\text { tonic and herbal tea, flavoring } \\
\text { agents (condiment and spice), } \\
\text { antiseptic, antitussive, and } \\
\text { carminative, as well as } \\
\text { treating colds }\end{array}$ & Mazandaran & {$[131]$} \\
\hline $\begin{array}{c}\text { Zataria multiflora } \\
\text { Boiss. }\end{array}$ & Avishane Shirazi & aerial parts & $\begin{array}{l}\text { immunostimulant, } \\
\text { antinociceptive, } \\
\text { anti-inflammatory, antioxidant, } \\
\text { antibacterial, antiviral, } \\
\text { antiparasitic, and antifungal }\end{array}$ & Kerman & {$[112,132-134]$} \\
\hline $\begin{array}{l}\text { Zhumeria majdae } \\
\text { Rech. f. \& } \\
\text { Wendelbo. }\end{array}$ & Mohrekhosh, & leaves & $\begin{array}{l}\text { stomach tonic, antiseptic } \\
\text { anti-nociceptive, and } \\
\text { anti-inflammatory }\end{array}$ & Hormozgan & {$[135,136]$} \\
\hline $\begin{array}{l}\text { Ziziphora capitata } \\
\text { Linnaeus. }\end{array}$ & kakuti-e Sarsan & aerial parts & $\begin{array}{l}\text { sedative, stomach tonic, } \\
\text { flatulence, common cold, } \\
\text { diarrhea, expectorant, coughing, } \\
\text { antiseptic, migraine, } \\
\text { and carminative }\end{array}$ & Kurdestan & {$[90,137]$} \\
\hline
\end{tabular}


Table 1. Cont.

\begin{tabular}{|c|c|c|c|c|c|}
\hline Scientific Name & $\begin{array}{l}\text { Common Name } \\
\text { (Vernacular Name) }\end{array}$ & Parts Used & $\begin{array}{l}\text { Therapeutic Effects \& Ethno } \\
\text { Pharmacological Properties } \\
\text { (The Number Inside } \\
\text { Paranthesis of Families Refers } \\
\text { to the Number of } \\
\text { Endemic Species) }\end{array}$ & Province(s) & Ref. \\
\hline $\begin{array}{c}\text { Ziziphora } \\
\text { clinopodioides Lam. }\end{array}$ & kakuti-e kuhi & aerial parts & $\begin{array}{l}\text { hypertension, sedative, } \\
\text { stomach, tonic, heart disorders, } \\
\text { common cold, inflammation, } \\
\text { depression, diarrhea, } \\
\text { expectorant, coughing, } \\
\text { antiseptic, migraine, } \\
\text { carminative, and } \\
\text { wound healing }\end{array}$ & $\begin{array}{c}\text { Mazandaran, Semnan, } \\
\text { Tehran, Kerman, } \\
\text { Chaharmahal va } \\
\text { Bakhtiari, Esfahan }\end{array}$ & {$[123,137]$} \\
\hline $\begin{array}{l}\text { Ziziphora persica } \\
\text { Bunge. }\end{array}$ & kakuti-e Irani & aerial parts & antimicrobial & $\begin{array}{l}\text { East and West } \\
\text { Azarbaijan }\end{array}$ & {$[137]$} \\
\hline $\begin{array}{l}\text { Ziziphora tenuior } \\
\text { Linnaeus. }\end{array}$ & Kakuti & aerial parts & antimicrobial & $\begin{array}{l}\text { Chaharmahal va } \\
\text { Bakhtiari }\end{array}$ & {$[112,137]$} \\
\hline \multicolumn{6}{|c|}{ Liliaceae (1) } \\
\hline $\begin{array}{l}\text { Lilium ledebouri } \\
\text { Boiss. }\end{array}$ & $\begin{array}{c}\text { Susan-e } \\
\text { Chelcheragh }\end{array}$ & corm, flower & $\begin{array}{l}\text { burns, injuries, inflammation, } \\
\text { and uterus disorders }\end{array}$ & Mazandaran, Gilan & {$[138]$} \\
\hline \multicolumn{6}{|c|}{ Rosaceae (3) } \\
\hline $\begin{array}{l}\text { Amygdalus } \\
\text { elaeagnifolia Spach. }\end{array}$ & Badame- Kermani & fruit, seed & $\begin{array}{l}\text { healing effects on skin damages } \\
\text { caused by radiotherapy, } \\
\text { anxiolytic properties, have a } \\
\text { decreasing effect on anxiety } \\
\text { and stress }\end{array}$ & $\begin{array}{c}\text { Lorestan, Arak, } \\
\text { Kohkilouyeh va } \\
\text { Boyer-Ahmad, } \\
\text { Chaharmahal va } \\
\text { Bakhtiari, Fars, Kerman }\end{array}$ & [139] \\
\hline $\begin{array}{l}\text { Amygdalus scoparia } \\
\text { Spach. }\end{array}$ & Badame Kohi & aerial parts & diabetes mellitus & Fars & {$[139]$} \\
\hline $\begin{array}{l}\text { Amygdalus lycioides } \\
\text { Spach. }\end{array}$ & Badame Vahshi & aerial parts & hyperlipidemia, hypoglycemia & $\begin{array}{l}\text { East and West } \\
\text { Azarbaijan, Lorestan, } \\
\text { Arak, Esfahan, Yazd, } \\
\text { Kerman, Tehran, } \\
\text { Hormozgan }\end{array}$ & {$[139]$} \\
\hline \multicolumn{6}{|c|}{ Scrophulariaceae (1) } \\
\hline $\begin{array}{c}\text { Verbascum } \\
\text { sublobatum Murb. }\end{array}$ & Gol-e Mahor & leaf & antioxidant & $\begin{array}{c}\text { Golestan, Mazandaran, } \\
\text { Tehran }\end{array}$ & {$[140,141]$} \\
\hline
\end{tabular}

\subsection{Endemic MAPs in Iran and the Plant Parts in Use}

Endemic medicinal plants are used for the relief of many disease conditions, namely respiratory system diseases, digestive system disorders, and muscular-skeletal system problems. The plant parts used for medicine (based on the species frequency) are aerial parts (55 spp.), flowers (17 spp.), roots (14 spp.), leaves (12 spp.), stems (9 spp.), fruits (7 spp.), seeds (5 spp.), corms (1 spp.), bulbs (1 spp.), pods (1 spp.), gums (1 spp.), resins (1 spp.), and manna (1 spp.) (Figure 3). The aerial parts are used in 55 out of 100 species. A total of 17 species are used for their flowers, which is followed by roots with 14 species (Table 1 and Figure 3). In many cases, more than one organ of the same species is used in the treatment of different maladies (Table 1). 
Table 2. A list of some neglected endemic MAPs of Iranian flora.

\begin{tabular}{|c|c|c|c|c|}
\hline \multirow{12}{*}{ Apiaceae } & \multirow{3}{*}{$\begin{array}{c}\text { Ferula } \\
\text { Ferulago }\end{array}$} & F. macrocolea. & \multicolumn{2}{|c|}{ F. stenocarpa } \\
\hline & & F. microcolea & \multicolumn{2}{|c|}{ F. behboudiana } \\
\hline & & \multicolumn{3}{|c|}{ F. phialocarpa } \\
\hline & Heracleum & \multicolumn{3}{|c|}{ H. nephrophyllum } \\
\hline & Pimpinella & \multicolumn{3}{|c|}{ P. tragioides } \\
\hline & Eryngium & \multicolumn{3}{|c|}{ E. bungei } \\
\hline & Anthemis & A. austroiranica. & A. kermanensis & A. odontostephana \\
\hline & \multirow{2}{*}{ Echinops } & E. aucheri & E. elymaticus & E. Iranshahrii \\
\hline & & E. lalesarensis & E. macrophyllus & \\
\hline & Helicrysum & H. oligocephalum & & \\
\hline & Scorzonera & S. subaphylla & & \\
\hline & Hertia & H. angustifolia & & \\
\hline Boraginaceae & Onosma & \multicolumn{3}{|c|}{ O. asperimum } \\
\hline Brassicaceae & Isatis & \multicolumn{3}{|c|}{ I. pachycarpa } \\
\hline Caryophyllaceae & Dianthus & \multicolumn{3}{|c|}{ D. macranthoides } \\
\hline \multirow{5}{*}{ Fabaceae } & \multirow{4}{*}{ Astragalus } & A. camptoceras & A. globiflorus & A. ovinus \\
\hline & & A. effusus & A. glaucacanthos & A. sieversianus \\
\hline & & A. ophiocarpus & A. crenatus & A. tribuloides \\
\hline & & A. mucronifolius & A. jolderensis & A. verus \\
\hline & Hedysarum & & H. persicum & \\
\hline Hypericaceae & Hypericum & & H. rechingeri & \\
\hline \multirow{11}{*}{ Lamiaceae } & \multirow{8}{*}{ Nepeta } & N. adenoclada & N. gloecocephala. & N. mirzayani \\
\hline & & N. allotria & N. hymenodonta & N. oxydonta \\
\hline & & N. archibaldii & N. iranshahrii & N. pogonosperma \\
\hline & & N. assurgens & N. koeieana & N. prostara \\
\hline & & N. assadii & N. chinophila & N. racemose \\
\hline & & N. bakhtiarica. & N. lasiocephala & N. scrophularioides \\
\hline & & N. eremokosmos & N. laxiflora & N. sessilifolia \\
\hline & & N. gedrosiaca & N. makuensis & N. straussii \\
\hline & Thymus & T. fedtschenkoi & \multicolumn{2}{|c|}{ T. migricus } \\
\hline & Hymenocrater & H. incanus & \multicolumn{2}{|c|}{ H. platystegius } \\
\hline & Stachys & \multicolumn{3}{|c|}{ S. ixodes } \\
\hline Malvaceae & Alcea & \multicolumn{3}{|c|}{ A. koelzii } \\
\hline Liliaceae & Fritillaria & F. kotschyana & \multicolumn{2}{|c|}{ F. zagrica } \\
\hline Linnaceae & Linum & \multicolumn{3}{|c|}{ L. persicum } \\
\hline Polygonaceae & Polygonum & \multicolumn{3}{|c|}{ P. aridum } \\
\hline Ranunculaceae & Clematis & \multicolumn{3}{|c|}{ C. ispahanica } \\
\hline \multirow{2}{*}{ Scrophulariaceae } & Scrophularia & \multicolumn{3}{|c|}{ S. farinosa } \\
\hline & Verbascum & & V. gabrielae & \\
\hline & Eremurus & & E. persicus & \\
\hline Xanthorrhoeaceae & Rheum & & R. persicum & \\
\hline & Rumex & & R. crispus & \\
\hline
\end{tabular}




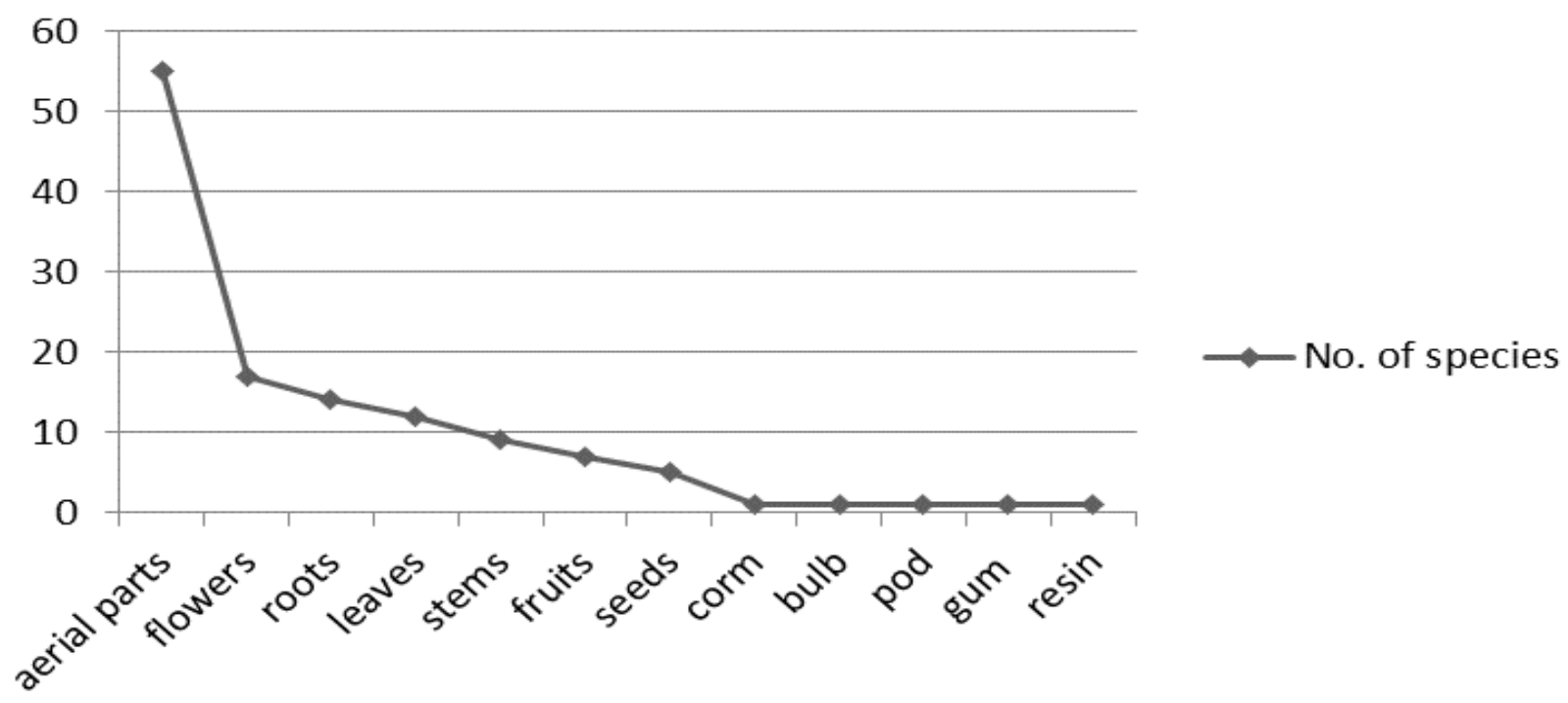

Figure 3. The plant parts of the Iranian endemic medicinal and aromatic plants in common use for curing the diverse maladies.

\subsection{Endemic MAPs Richness across Iran}

The distribution of Iranian endemic MAPs is shown in Figures 4-6. As seen on the maps, the endemic MAPs of Iranian flora are distributed in nearly all parts of the country. Most of these valuable plants flourish in small populations in mountainous habitats. In Southwest Asia in general and in Iran in particular, mountains have the dominant role in the development of endemic species [14].

Endemic richness in Iran is significantly related to the topography and the climatic conditions of the regions and numerous mountain ranges [18]. There are many mountain peaks in Iran with an elevation higher than $4000 \mathrm{~m}$ [15], and signficant richness was reported in high mountains (Sabalan Mts., Sahand Mts., Talysh Mts., Shahu Mts., Alvand., Oshtorankuh Mts., Zardkuh Mts., Dena Mts., Alvand Mts., Karkas Mts., Binalood Mts., Shirkuh Mts., and Hezar-Lalezar Mts.) [1].

Most of the published information on the number of endemic MAPs of Iran is based on the political units, such as provinces. The number of endemic MAPs varies greatly among 31 provinces (from 0 in Qom to 25 in Esfahan) (Figure 4). Most of them are distributed in the main mountain ranges of Esfahan, Kerman, Fars, Tehran, Chaharmahal va Bakhtiari, East Azarbaijan, Lorestan, West Azarbaijan, Hamadan, and Mazandaran provinces, respectively (Figure 4).

Noroozi et al. (2018) reported five AEs from Iran, (i.e., Zagros, Azarbaijan, Kopet DaghKhorassan, Alborz, and Central Alborz) (Figure 5). The ten provinces mentioned above are predominantly located in these five AEs (Figure 5), which are in the Iran-o-Turanian region, one of the three major phytogeographic regions in Iran (i.e., the Saharo-Sindian, the Iran-o-Turanian, and the Euro-Siberian) (Figure 6). The Iran-o-Turanian region composes the highest percentage (68.29\%) of endemic MAPs in Iran (Figure 6) and is considered as an essential area of endemism in Asia [1,12,15].

Among AEs of Iran, Zagros was found to host the maximum number of endemic MAPs with 125 species, followed by Azarbaijan (46 species), Alborz, and Central Alborz (40 species), and Kopet Dagh-Khorassan (4 species) (Figure 5). The least number of endemic MAPs was found in Kopet Dagh-Khorassan with only four species. The low number of endemic MAPs in Kopet Dagh-Khorassan results from the limited size of the area. The mountainous areas of Kopet Dagh-Khorassan do not expand beyond Iran except for a small section in the north [1]. 


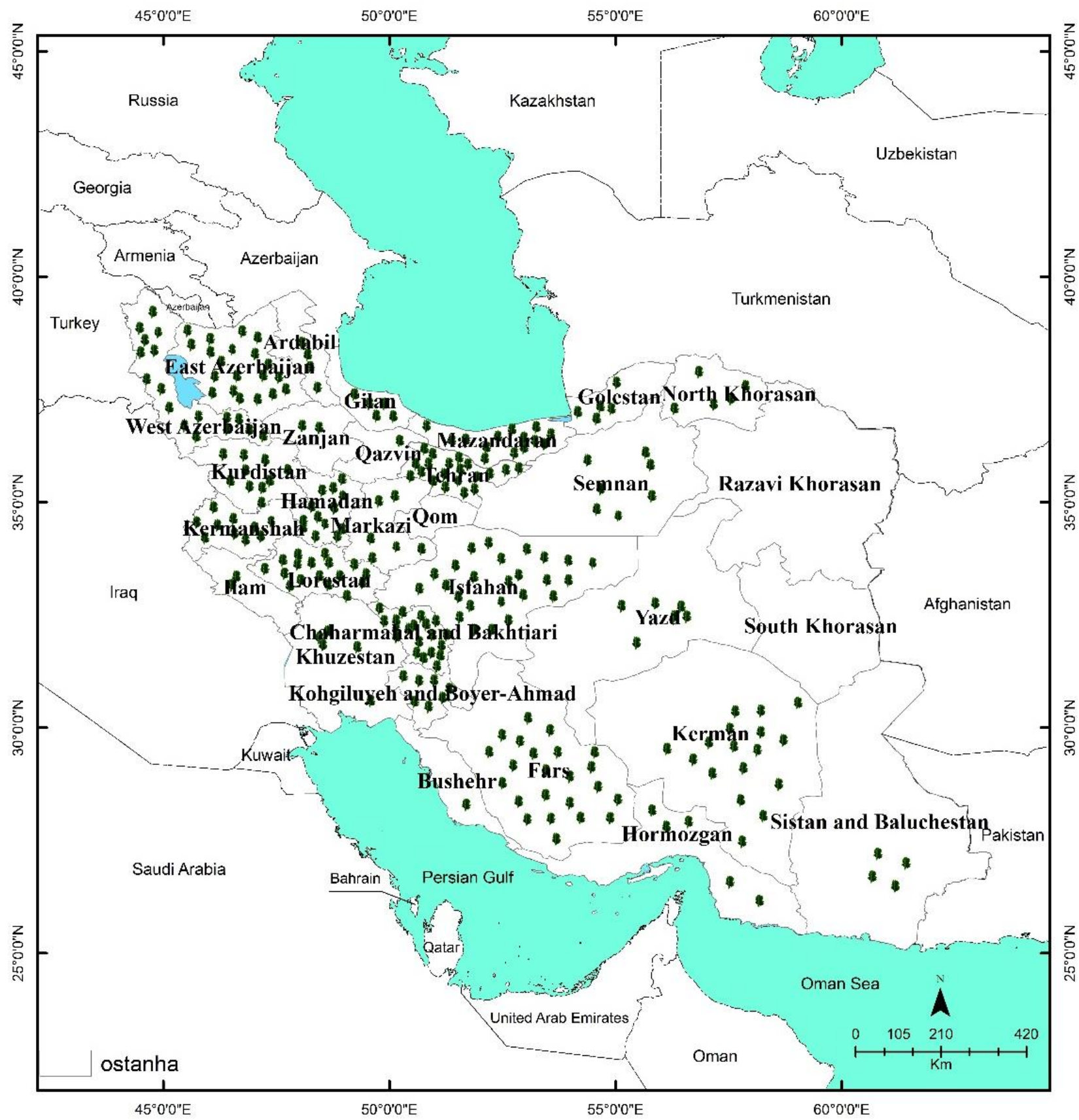

Figure 4. The distribution pattern of Iranian endemic medicinal and aromatic plants across the provinces.

Five AEs (Alborz, Central Alborz, Zagros, Azarbaijan, and Kopet Dagh-Khorassan) and three phytogeographic regions (the Iran-o-Turanian, the Saharo-Sindian, and the EuroSiberian) in Iran cover parts of two global BHs (i.e., Irano-Anatolian and Caucasus), and it is estimated that $97 \%$ of the endemic vascular plant species of this country are limited to these sectors [12]. The Caucasus hotspot includes Georgia, Azarbaijan, and a small portion of northern Iran, and the Iran-Anatolian hotspot includes significant parts of northern and western Iran, central and eastern Turkey, a small portion of southern Georgia, the Nakhchivan province of the country of Azerbaijan, much of Armenia, northeastern Iraq, and the northern Kopet Dagh range in Turkmenistan (Figure 7). 


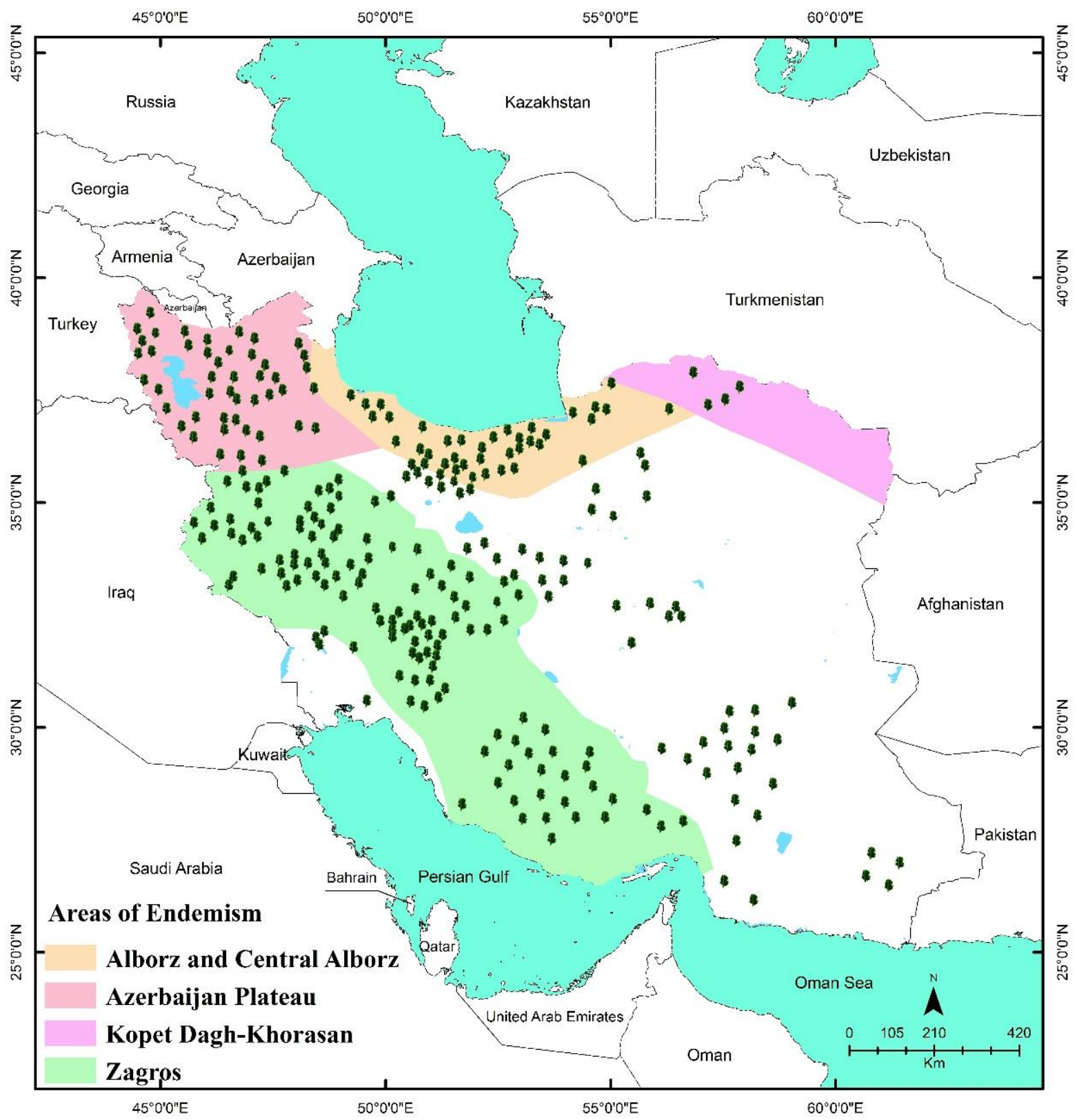

Figure 5. Distribution patterns of the endemic medicinal and aromatic plants of Iranian flora by endemism area.

Our findings on the distribution pattern of endemic MAPs in Iran is similar to the results of a study at the University of Vienna about the distribution of the Asteraceae family as a model group in Iran [14], a study at the University of Tehran about the distribution of Iranian trees and shrubs [19], and a study about the biodiversity and floristic endemism of Fritillaria spp. in Iran [13]. Our results verify the vast distribution pattern for a large number of species across the country, and even clarify the diversification of many unconsidered species with medicinal values that have not been the focus of former studies. 


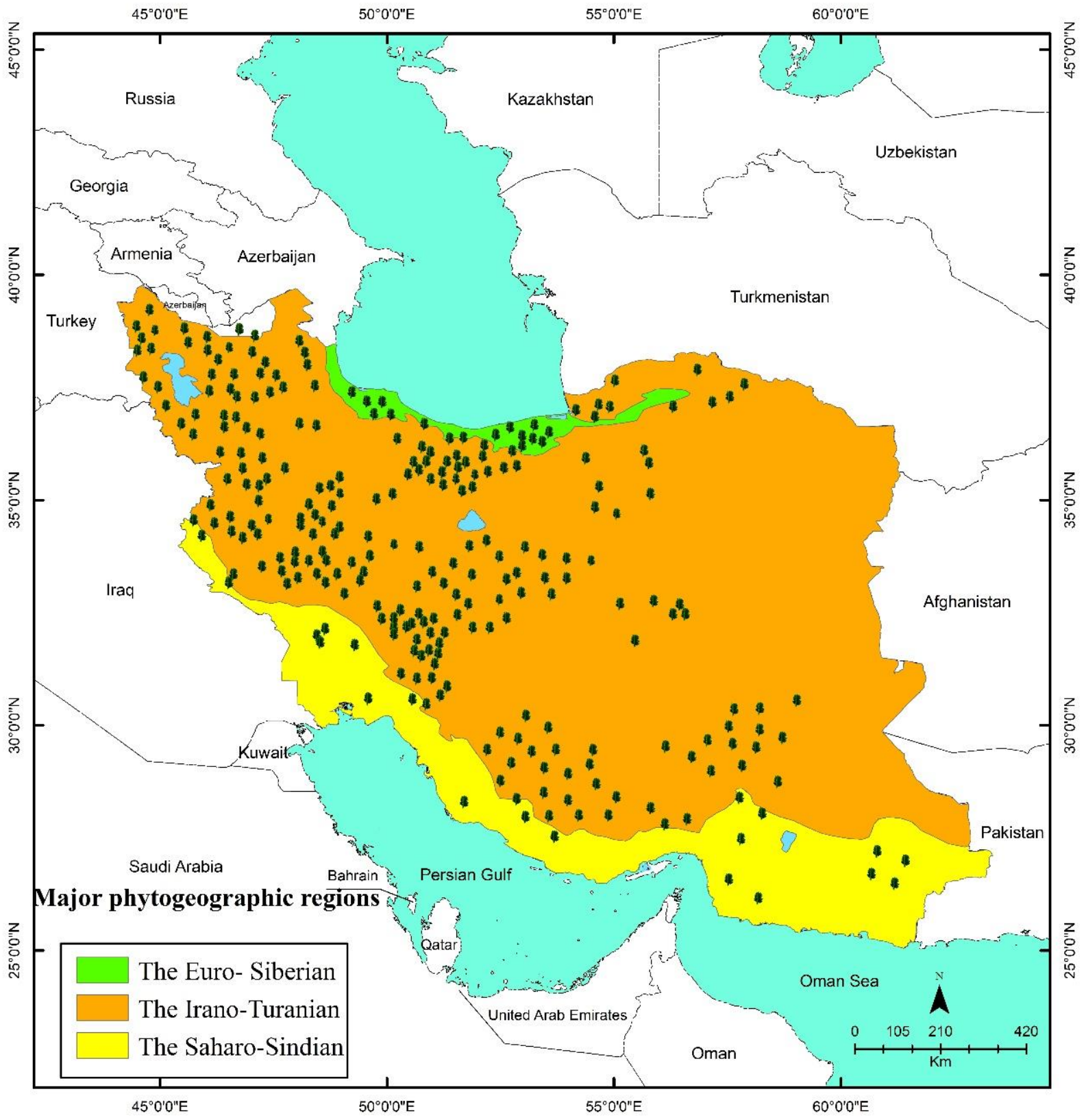

Figure 6. Topogeographic map of Iran indicating phytogeographical regions and distribution of endemic medicinal and aromatic plants.

\subsection{Relationship between the Climate and Richness of Endemic MAPs of Iran}

Iran, as a vast country with 31 political provinces, has significant cross-sectional variation in the climate types and is characterized by different climates ranging from arid to semi-arid mountain ranges. Various climates of Iran include arid, semi-arid, hyper-arid, humid, semi-humid, highly-humid, and Mediterranean [17] (Figure 8). Thus, due to the particular distribution of endemic MAPs and different climates in Iran, we investigated how endemic MAPs correlate with the different climate conditions in this country. 


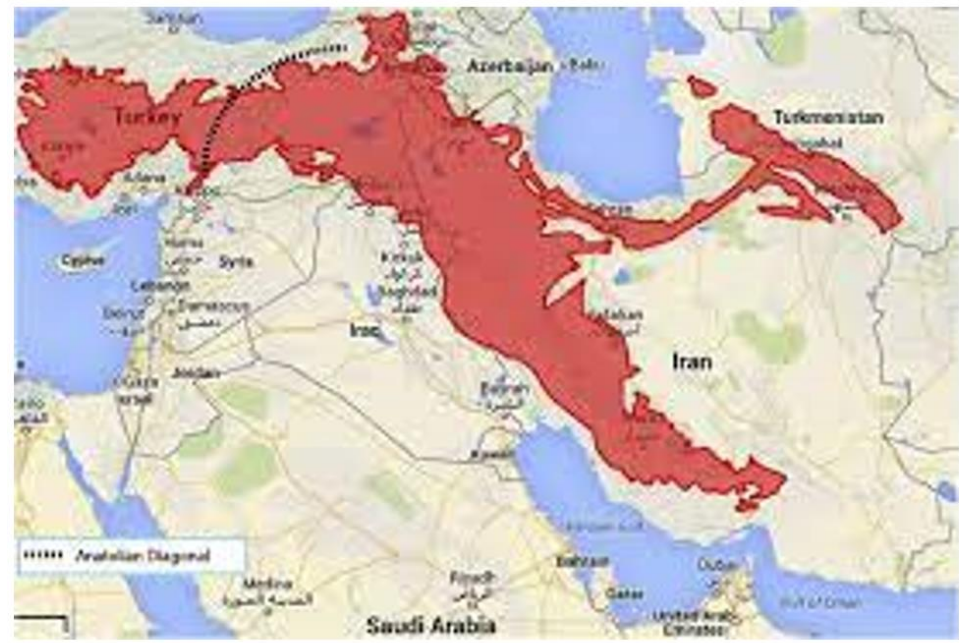

(A)

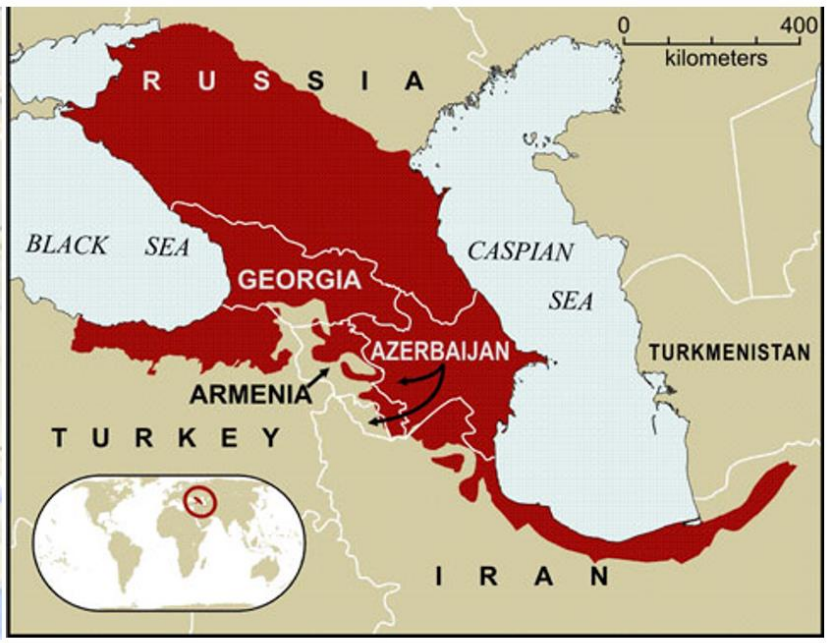

(B)

Figure 7. Two biodiversity hotspots regions of Iran (adopted from https:/ / biodiversity.doe.ir/portal/ home/?778961/ (accessed on 26 January 2022). Note: (A) shows Irano-Anatolian biodiversity hotspot, and (B) shows the Caucasus biodiversity hotspot.

As shown in Figure 8, the climate of one province is mainly different from that of another. The provinces we studied were found to host a diverse range of endemic MAPs, dominantly belonging to five different climate conditions, i.e., semi-arid, humid, semihumid, highly-humid, and Mediterranean. The maximum number of species ( $86 \mathrm{spp}$.) were found in the semi-arid climate, followed by the humid (28 spp.), highly-humid (27 spp.), semi-humid (14 spp.), and the Mediterranean (12 spp.) climates (Figure 8). Overlapping of the species within the areas has been observed (Figure 8).

Like all other species of the biosphere, MAPs have no exemption from the effects of climate change [27], especially some MAPs that are endemic to the geographic regions which are more at risk and vulnerable to climate changes [28]. Climate change is attributable directly or indirectly to the human enterprises that alter atmospheric composition [28]. There is a high risk of mass extinction of biodiversity as the planet warms and the climate changes, and Iran is also impacted by climate change, especially due to the presence of mountains and near-desert areas. For example, some cold-adapted MAPs in mountainous hillsides in Iran have begun to gradually migrate higher up mountain summits, a phenomenon correlated with climate warming [15]. Eventually, this migration of MAPs may cause them to face extinction [15]. Higher temperatures and lower water availability can cause climate changes that likely have a significant impact on MAPs growth in the near future [29].

Climate change will alter the environmental conditions for MAPs, especially in arid and semi-arid regions. In other words, some regions in the "Humid" class may be turned into the "Arid" class, and some regions that are currently "Semi-Arid" may be turned into the "Hyper-Arid" class regarding the climatic change [17].

Some MAPs are drought-tolerant, and the stress may cause increases in the concentration of their secondary metabolites (either by decreasing biomass or by increasing the actual production of the metabolites). For other species, however, relationships with specific pollinators may be disrupted by the phenological alterations arising from climate change [27]. 


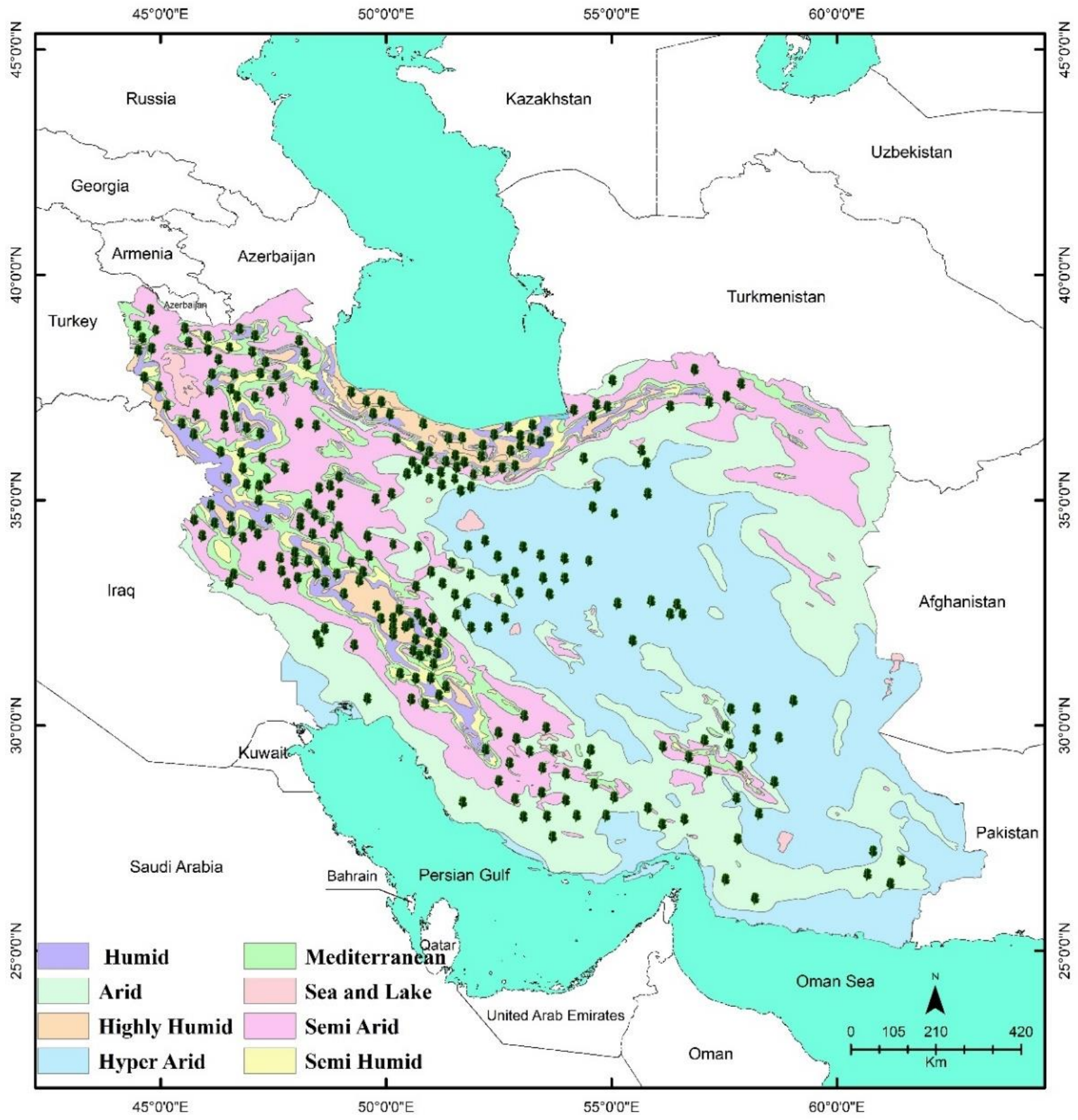

Figure 8. Climate map of Iran showing the location of endemic medicinal and aromatic plants.

\section{Need for Conservation}

The distribution of the MAPs on the Earth is not uniform and differs in different geographical regions. Regardless, they serve a considerable role in the health care of people across the world. As per the data available, more than $75 \%$ of the world's population relies mainly on medicinal plants and herbal medicines for their health care needs $[6,8,9]$.

The geographic distribution and biological attributes of these kinds of plants must be known to guide conservation programs and better manage our use of the biosphere [6]. Human management must be able to balance the competing demands of obtaining the greatest resources for the present generation while preserving the potential for future generations. In the preservation approach, all species are not of equal significance, and setting priorities is the most important step in the conservation programs [4]. In this context, 
endemic MAPs with limited distribution are of greater importance than exotic species with a wide distribution.

The geographic distribution of endemic MAPs must be known to guide the conservation proceedings, e.g., to assess whether species protection should take place in nature or a greenhouse [6]. Two sets of suggestions relating to the conservation of endemic MAPs have been developed as follows: in situ (protection of species in their natural environment) and ex situ (protection of species outside their natural habitat) [6,9]. Both conservation strategies (i.e., in situ and ex situ) and also resource management (e.g., good agricultural practices and sustainable use solutions) should be adequately taken into account for the sustainable use of MAPs resources [6].

Nowadays, biotechnological tools, such as micropropagation, tissue culture, synthetic seed technology, and molecular markers, provide new and complementary options for plant conservation, including short-, medium-, and long-term strategies, and their application for plant species conservation has considerably increased [30]. Indeed, no conservation strategy alone may be sufficient to prevent species from extinction. Therefore, it is important to combine conservation strategies (in situ, ex situ, biotechnological tools, and so on) that will complement each other in the effort to preserve a given species.

\section{Perspectives for Conservation and Habitat Management of MAPs}

In summary, while MAPs have contributed to the healthcare systems and economy of rural populations, the following critical issues should be addressed:

1. The national and international demand for MAPs in Iran is increasing, which creates tremendous pressure on natural habitats. There is no formal harvesting system, so little is known about which plants are being harvested, from where, and in what quantity. There is a clear need for a system of monitoring and tracking wild harvesting, specifically in the five AEs.

2. To reduce the harsh harvesting pressure on wild populations and to conserve the vulnerable species, novel technologies should be introduced to improve the culture, harvesting, and drying of the MAPs, especially the value-added endemic medicinal and aromatic plants.

3. The programmed utilization management of the endemic MAPs can generate more income for the farmers and local harvesters and, therefore, protects the environment and MAPs populations from the excessive harvests and possible extinction.

4. In addition to the efforts for mitigating the extinction of endemic MAPs, the preservation of traditional knowledge is a component of conservation. Folk understanding of medicinal and aromatic plants used by the inhabitants of Iran should be recorded, particularly in the rural areas of the country, where there is no or limited access to hospitals, drugstores, and health experts.

5. Most of the published information on the number of endemic MAPs of Iran is related to the political provinces. Many large provinces in the arid or hyper-arid regions are relatively poor in endemic MAPs, while many provinces in the humid, highlyhumid, semi-humid, semi-arid, and Mediterranean regions are extraordinarily rich in endemic MAPs. Consequently, studying the environmental potentials and risks of these provinces would protect the majority of the endemic plants from excessive harvest regimes and the possible extinction.

6. Although, species conservation, especially that of the endangered species, may be more effective through natural habitat (in situ conservation) inspections and environmental managements; the ex-situ techniques also can be used to complement the in-situ methods. Biotechnological methods such as micropropagation, tissue culture, synthetic seed technology, and molecular marker approaches can be used to amend the product and alter the efficacy of medicinal and aromatic plants.

7. Supplementary studies can be carried out in other fields such as phylogenetic diversity or DNA barcoding to emphasize the importance of the endemic MAPs, the AEs, and the conservation programs. 
8. $16.7 \%$ of Earth's land surface is home to $77 \%$ of all endemic plant species representing $36 \mathrm{BHs}$ in the world, and Iran holds two of the biodiversity hotspots. So, to conserve Iran's biodiversity, the Iranian government should launch projects to conduct biodiversity investigation across the country, especially in endemic MAPs and specifically in Tehran, Esfahan, Kerman, Fars, Chaharmahal va Bakhtiari, East Azrbaijan, Lorestan, West Azarbaijan, Hamadan, and Mazandaran provinces, to more thoroughly understand their distribution, abundance, and ecology for the long-term and sustainable production of major MAPs.

9. By describing the geographical distribution and ecological aspects of the endemic MAPs along with a concentrated insight into their biodiversity concerning their taxonomic status, a more obvious understanding of the essential elements of conservation strategies can be supplied for all the involved preservationists, government sectors, and NGOs.

\section{Conclusions}

Iran is a country of diverse landforms, climates, and species of MAPs (2300 out of 8200 species). Of the $36 \mathrm{BHs}$ recognized in the world, Iran has two significant hotspots-the Irano-Anatolian and the Caucasus. Reviewing the phytogeographical distribution pattern of MAPs reveals that the Iran-o-Turanian region is the main center of diversity for the Iranian endemic MAPs. Considering the total number of AEs recognized in Iran (Alborz and Central Alborz, Zagros, Azarbaijan, and Kopet Dagh-Khorassan), the number of endemic MAPs will be interesting. Our data show the density of endemism in Esfahan, Kerman, Fars, Tehran, Chaharmahal va Bakhtiari, East-Azarbaijan, Lorestan, West-Azarbaijan, Hamadan, and Mazandaran provinces is higher than in the other provinces of Iran. The dominant MAPs diversity harboring localities of Iran were found to host a diverse range of endemic MAPs (100 species of medicinal and aromatic importance, from 10 families and 30 genera, with potential uses for therapeutic purposes). Although all of Iran is essential for conservation, those areas rich in endemic MAPs that are prone to climate change, are relatively more significant to consider their diversity inspection and further conservation programs. This review article provides the policy-makers baseline data to make suitable decisions for the conservation of endemic medicinal and aromatic plants at the national and provincial levels. With an increasing world population and climate change, the identification of all $\mathrm{BHs}$ at a finer scale and identifying AEs of every country are essential elements for the execution of global conservation management programs.

Author Contributions: Conceptualization, M.B.H. and H.G.; data curation, M.B.H., H.G. and M.E.; methodology, M.B.H., H.G. and M.E.; visualization, M.B.H., H.G., M.E. and M.H.A. writing-original draft, H.G. and M.B.H.; writing-review and editing, M.B.H., H.G., M.H.A., M.S. and S.D. All authors have read and agreed to the published version of the manuscript.

Funding: This research received no external funding.

Institutional Review Board Statement: Not applicable.

Informed Consent Statement: Not applicable.

Data Availability Statement: All-new research data were presented in this contribution.

Conflicts of Interest: The authors declare that they have no conflict of interest.

\section{References}

1. Noroozi, J.; Talebi, A.; Doostmohammadi, M.; Manafzadeh, S.; Asgarpour, Z.; Schneeweiss, G. Endemic diversity and distribution of the Iranian vascular flora across phytogeographical regions, biodiversity hotspots and areas of endemism. Sci. Rep. 2019, 9, 12991. [CrossRef] [PubMed]

2. Hrdina, A.; Romportl, D. Evaluating global biodiversity hotspots-Very rich and even more endangered. J. Landsc. Ecol. 2017, 10, 108-115. [CrossRef]

3. Myers, N.; Mittermeier, R.; Mittermeier, C.; Fonseca, G.; Kent, J. Biodiversity hotspot for conservation priorities. Nature 2000, 403, 853-858. [CrossRef] [PubMed] 
4. Gonçalves-Souza, D.; Verburg, P.H.; Dobrovolski, R. Habitat loss, extinction predictability and conservation efforts in the terrestrial ecoregions. Biol. Conserv. 2020, 246, 108579. [CrossRef]

5. Hobohm, C.; Janišová, M.; Steinbauer, M.; Landi, S.; Field, R.; Vanderplank, S.; Beierkuhnlein, C.; Grytnes, J.-A.; Vetaas, O.R.; Fidelis, A.; et al. Global endemics-area relationships of vascular plants. Perspect. Ecol. Conserv. 2019, 17, 41-49. [CrossRef]

6. Chen, S.; Yu, H.; Luo, H.-M.; Wu, Q.; Li, C.-F.; Steinmetz, A. Conservation and sustainable use of medicinal plants: Problems, progress, and prospects. Chin. Med. 2016, 11, 37. [CrossRef]

7. Wangchuk, P.; Tobgay, T. Contributions of medicinal plants to the gross national happiness and biodiscovery in bhutan J. Ethnobiol. Ethnomed. 2015, 11, 48. [CrossRef]

8. Astutik, S.; Pretzsch, J.; Ndzifon Kimengsi, J. Asian medicinal plants' production and utilization potentials: A review. Sustainability 2019, 11, 5483. [CrossRef]

9. Kumar, A.; Jnanesha, A.C. Conservation of rare and endangered plant species for medicinal use. Int. J. Sci. Res. 2016, 5, 1370-1372

10. Salmerón-Manzano, E.; Garrido-Cardenas, J.A.; Manzano-Agugliaro, F. Worldwide research trends on medicinal plants. Int. J. Environ. Res. Public Health 2020, 17, 3376. [CrossRef]

11. Kidane, Y.O.; Steinbauer, M.J.; Beierkuhnlein, C. Dead end for endemic plant species? A biodiversity hotspot under pressure. Glob. Ecol. Conserv. 2019, 19, e00670. [CrossRef]

12. Noroozi, J.; Naqinezhad, A.; Talebi, A.; Doostmohammadi, M.; Plutzar, C.; Rumpf, S.B.; Asgarpour, Z.; Schneeweiss, G.M Hotspots of vascular plant endemism in a global biodiversity hotspot in Southwest Asia suffer from significant conservation gaps. Biol. Conserv. 2019, 237, 299-307. [CrossRef]

13. Kiani, M.; Mohammadi, S.; Babaei, A.; Sefidkon, F.; Naghavi, M.R.; Ranjbar, M.; Razavi, S.A.; Saeidi, K.; Jafari, H.; Asgari, D.; et al. Iran supports a great share of biodiversity and floristic endemism for Fritillaria spp. (Liliaceae): A review. Plant Divers. 2017, 39, 245-262. [CrossRef]

14. Noroozi, J.; Talebi, A.; Doostmohammadi, M.; Rumpf, S.; Linder, H.; Schneeweiss, G. Hotspots within a global biodiversity hotspot-Areas of endemism are associated with high mountain ranges. Sci. Rep. 2018, 8, 10345. [CrossRef] [PubMed]

15. Noroozi, J.; Akhani, H.; Breckle, S.-W. Biodiversity and phytogeography of the alpine flora of Iran. Biodivers. Conserv. 2013, 17, 493-521. [CrossRef]

16. Sotoudeh Foumani, B.; Rostami Shahraji, T.; Mohammadi Limaei, S. Role of political power in forest administration policy of Iran. Casp. J. Environ. Sci. 2017, 15, 181-199. [CrossRef]

17. Zarei, A. Evaluation of changes in different climates of iran, using de martonne index and mann-kendall trend test. Nat. Hazards Earth Syst. Sci. Discuss. 2014, 2, 2245-2261. [CrossRef]

18. Mahmoudi, S.; Khoramivafa, M.; Hadidi, M. Investigation of the relationship between altitude and aspect with plant diversity: A case study from nawa mountain ecosystem in Zagros, Iran. J. Rangel. Sci. 2018, 8, 129-142.

19. Mehrabian, A.R.; Sayadi, S.; Majidi Kuhbenani, M.; Hashemi Yeganeh, V.; Abdoljabari, M. priorities for conservation of endemic trees and shrubs of Iran: Important Plant Areas (IPAs) and Alliance for Zero Extinction (AZE) in SW Asia. J. Asia-Pac. Biodivers. 2020, 13, 295-305. [CrossRef]

20. Buso, P.; Manfredini, S.; Reza Ahmadi-Ashtiani, H.; Sciabica, S.; Buzzi, R.; Vertuani, S.; Baldisserotto, A. Iranian medicinal plants: From ethnomedicine to actual studies. Medicina 2020, 56, 97. [CrossRef]

21. Jalilvand Djalilvand, H.; Sabet Teimouri, M. Medicinal \& aromatic plants ethno botanical survey of herbal remedies traditionally used in North Khorasan Province of Iran. Med. Aromat. Plants 2015, 4, 2015.

22. Sheibani, M.; Nayernouri, T.; Dehpour, A.R. Herbal medicines and other traditional remedies in Iran-A tragedy unfolds. Arch. Iran. Med. 2018, 21, 312-314. [PubMed]

23. Mozaffarian, V. Dictionary of Iranian Plant Names: Latin, English, Persian; Farhang Moaser Publication: Tehran, Iran, 1998; ISBN 978-9645545404.

24. Jalili, A. Red Data Book of Iran: A Preliminary Survey of Endemic, Rare E Endangered Plant Species in Iran; Research Institute of Forests and Rangelands: Teheran, Iran, 1999; ISBN 978-964-473-061-0.

25. Ziarati, P.; Asgarpanah, J.; Sarabiyan Nejad, S. Essential oil of nepeta genus (lamiaceae) from Iran: A review. J. Essent. Oil Res. 2013, 26, 1-12. [CrossRef]

26. Ibrahim, A.; ALI, M. Constituents of nepeta crassifolia (Lamiaceae). Turk. J. Chem. 2007, 31, 467-470.

27. Applequist, W.L.; Brinckmann, J.A.; Cunningham, A.B.; Hart, R.E.; Heinrich, M.; Katerere, D.R.; van Andel, T. Scientists' warning on climate change and medicinal plants. Planta Med. 2020, 86, 10-18. [CrossRef] [PubMed]

28. Das, M.; Jain, V.; Malhotra, S. Impact of climate change on medicinal and aromatic plants: Review. Indian J. Agric. Sci. 2016, 86, 1375-1382.

29. Tangjitman, K.; Wongsawad, C.; Winijchaiyanan, P.; Sukkho, T.; Kamwong, K.; Pongamornkul, W.; Trisonthi, C. Traditional knowledge on medicinal plant of the Karen in Northern Thailand: A comparative study. J. Ethnopharmacol. 2013, 150, 232-243. [CrossRef]

30. Coelho, N.; Gonçalves, S.; Romano, A. Endemic plant species conservation: Biotechnological approaches. Plants 2020, 9, 345 [CrossRef]

31. Aleebrahim-Dehkordy, E.; Ghasemi Pirbalouti, A.; Mirhoseini, M. A comprehensive review on Allium hirtifolium boiss as a medicinal and edible plant. Der Pharm. Lett. 2016, 8, 188-196. 
32. Asgarpanah, J.; Ghanizadeh, B. Pharmacologic and medicinal properties of Allium hirtifolium boiss. Afr. J. Pharm. Pharmacol. 2012, 6, 1809-1814. [CrossRef]

33. Naeini, A.; Yaraee, R.; Shokri, H. Antifungal and immunomodulatory activity of Allium jesdianum boiss extracts. J. Herbmed. Pharmacol. 2019, 9, 75-80. [CrossRef]

34. Zandpour, F.; Vahabi, M.; Allafchian, A.; Farhang, H. Phytochemical investigation of the essential oils from the leaf and stem of Dorema ammoniacum d. don. (apiaceae) in Central Zagros, Iran. J. Herb. Drugs 2016, 7, 109-116.

35. Mianabadi, M.; Hoshani, M.; Salmanian, S. Antimicrobial and anti-oxidative effects of methanolic extract of Dorema aucheri boiss J. Agric. Sci. Technol. 2015, 17, 623-634.

36. Shahabipour, S.; Firuzi, O.; Asadollahi, M.; Faghimirzaei, E.; Javidnia, K. Essential oil composition and cytotoxic activity of Ducrosia anethifolia and Ducrosia flabellifolia from Iran. J. Essent. Oil Res. 2012, 25, 160-163. [CrossRef]

37. Karami, A.; Bohlooli, A. Essential oil chemical diversity of Ducrosia anethifolia (dc.) boiss. Accessions from Iran. J. Essent. Oil Bear. Plants 2017, 20, 1342-1348. [CrossRef]

38. Mostafavi, A.; Afzali, D.; Mirtadzadini, M. Chemical composition of the essential oil of Ducrosia anethifolia (dc.) boiss. from kerman province in Iran. J. Essent. Oil Res. 2008, 20, 509-512. [CrossRef]

39. Mostafavi, A.; Shamspur, T.; Afazali, D.; Mirtadzadini, M. Chemical composition of the essential oil of Ducrosia assadii alava. from Kerman Province in Iran. J. Essent. Oil Res. 2010, 22, 300-302. [CrossRef]

40. Assadipour, A.; Sharififar, F.; Robati, M.; Samzadeh, V.; Esmaeilpour, K. Composition and antioxidant effect of the essential oils of the flowers and fruits of Ducrosia assadii alava., a unique endemic plant from Iran. J. Biol. Sci. 2013, 13, 288-292. [CrossRef]

41. Rahimi-Nasrabadi, M.; Gholivand, M.B.; Niasari, M.; Vatanara, A. Chemical composition of the essential oil from aerial parts of echinophora Platyloba DC. from Iran. J. Med. Plants 2010, 9, 53-56.

42. Jelodarian, Z.; Mansourian, M.; Shokoohinia, Y. Phytochemical investigation of acetone extraction of echinophora cinerea aerial parts. Res. J. Pharmacogn. 2017, 14, 117.

43. Bazvandi, L.; Shokoohinia, Y.; Ghiasvand, N.; Mohajeri, P.; Ashrafi, B.; Salimikia, I. The antimicrobial activity of different extracts from Echinophora Platyloba DC. Herb. Med. J. 2017, 2, 153-157. [CrossRef]

44. Hamah, A.B. Chemical Composition and bioactivity of isolated components of echinophora platyloba, a medicinal plants find in Kurdistan Region of Iraq, using Gc-Ms, Nmr and Mtt bioassay method. Int. J. Sci. Res. 2015, 4, 19.

45. Asghari, G.; Sajjadi, S.E.; Hasan, S.; Kh, Y. Essential oil constituents of Echinophora Platyloba DC. Iran. J. Pharm. Res. 2003, 2, 185-186.

46. Mohammadhosseini, M.; Venditti, A.; Sarker, S.D.; Nahar, L.; Akbarzadeh, A. The genus ferula: Ethnobotany, phytochemistry and bioactivities-A review. Ind. Crops Prod. 2019, 129, 350-394. [CrossRef]

47. Sahebkar, A.; Iranshahi, M. Biological activities of essential oils from the Genus Ferula (Apiaceae). Asian Biomed. 2010, 4, 835-847. [CrossRef]

48. Sattar, Z.; Iranshahi, M. Phytochemistry and pharmacology of Ferula persica boiss.: A review. Iran. J. Basic Med. Sci. 2017, 20, 18. [CrossRef]

49. Shahverdi, A.R.; Iranshahi, M.; Mirjani, R.; Jamalifar, H.; Amin, G. Bioassay-guided isolation and identification of an antibacterial compound from ferula persica var. persica roots. DARU 2005, 13, 17-19.

50. Shamasbi, L.R.; Razavi, S.M.; Chaparzadeh, N. The study of secondary metabolic of ferulago carduchorum boiss \& hausskn. Nat Prod. Indian J. 2018, 14, 120

51. Golfakhrabadi, F.; Khanavi, M.; Ostad, S.N.; Saeidnia, S.; Vatandoost, H.; Abai, M.R.; Hafizi, M.; Yousefbeyk, F.; Rad, Y.R.; Baghenegadian, A.; et al. Biological activities and composition of ferulago carduchorum essential oil. J. Arthropod Borne Dis. 2015, 9, 104-115.

52. Mohebat, R.; Mosslemin, M.H.; Masoudi, S.; Dad, V.; Rustaiyan, A. Composition and antibacterial activity of the essential oils from aerial parts, stems, flowers and leaves of Ferulago Contracta From Iran. J. Essent. Oil Bear. Plants 2010, 13, 607-614. [CrossRef]

53. Radjabian, T.; Salimi, A.; Rahmani, N. Essential-oil composition of the fruits of six Heracleum L. species from iran:chemotaxonomic significance. Chem. Biodivers. 2014, 11, 1945-1953. [CrossRef]

54. Torbati, M.; Nazemiyeh, H.; Lotfipour, F.; Asnaashari, S.; Nemati, M.; Fathiazad, F. Composition and Antibacterial activity of heracleum transcaucasicum and heracleum anisactis aerial parts essential oil. Adv. Pharm. Bull. 2013, 3, 415-418. [CrossRef] [PubMed]

55. Mazandaranim, M.; Makari, S.; Bajian, G.; Zarghami Moghaddam, P.; Abroudi, M. Evaluation of Phytochemical and antioxidant activity in different parts of heracleum gorganicum Rech.F. in Golestan Province of Iran. Iran. J. Plant Physiol. 2011, 2, 381-386.

56. Mirza, M.; Najafpoor Navaei, M.; Behrad, Z. Chemical composition of essential oil from leaves, stems, flowers and seeds of heracleum rechingeri manden. from Iran. J. Med. Plants Prod. 2015, 4, 99-102. [CrossRef]

57. Ghasemi, M.; Kaini, S. The essential oil composition of wild celery (kelussia odoratissima mozaff.): An iranian endemic plant. Adv. Herb. Med. 2016, 2, 21-27.

58. Ghasemi Pirbalouti, A.; Aghaee, K.; Kashi, A.; Malekpoor, F. Chemical composition of the essential oil of wild and cultivated plant populations of kelussia odoratissima mozaff. J. Med. Plants 2012, 6, 449-454. [CrossRef]

59. Hossaini, N.; Salehi-Arjmand, H.; Ghorbanpour, M. Phytochemical composition of the essential oils from two pimpinella 1. species (p. deverroides boiss. and p. tragium vill.) growing wild in Iran. J. Med. Plants Prod. 2014, 3, 47-51. 
60. Moradalizadeh, M.; Salajeghe, M.; Mehrabpanah, M. Chemical characterization of the essential oil of prangos cheilanthifolia from Iran. Chem. Nat. Compd. 2015, 51, 573-574. [CrossRef]

61. Farhang Sardrodi, A.; Soleymani, A.; Kheiry, A. Essential oil composition of Achillea aucheri boiss at different growing altitudes in Damavand, Iran. J. Agric. Sci. Technol. 2018, 19, 357-364.

62. Afshari, M.; Rahimmalek, M. Variation in essential oil composition, bioactive compounds, anatomical and antioxidant activity of achillea aucheri, an endemic species of Iran, at different phenological stages. Chem. Biodivers. 2018, 15, e1800319. [CrossRef]

63. Mohammadhosseini, M.; Sarker, S.D.; Akbarzadeh, A. Chemical Composition of the essential oils and extracts of achillea species and their biological activities: A review. J. Ethnopharmacol. 2017, 199, 257-315. [CrossRef] [PubMed]

64. Varasteh-kojourian, M.; Abrishamchi, P.; Matin, M.M.; Asili, J.; Ejtehadi, H.; Khosravitabar, F. Antioxidant, cytotoxic and DNA protective properties of achillea Eriophora DC. and achillea biebersteinii afan. extracts: A comparative study. Avicenna J. Phytomed. 2017, 7, 157-168. [PubMed]

65. Dehghan, G.; Elmi, F. Essential oil combination of three species of achillea growing wild in east azarbayjan-Iran. Adv. Herb. Med. 2015, 1, 22-28.

66. Maghsoodlou, M.; Valizadeh, J.; Mottaghipisheh, J.; Rahneshan, N. Evaluation of the essential oil composition and antioxidant activity of achillea eriophora as a medicinal plant. J. Med. Herbs 2015, 5, 187-192.

67. Ghani, A.; Azizi, M.; Hassanzadeh, M.; Pahlavanpour, A. Essential oil composition of achillea eriophora, A. nobilis, A. biebersteinii and a. wilhelmsii from Iran. J. Essent. Oil Bear. Plants 2008, 11, 460-470. [CrossRef]

68. Dastjerdi, L.; Mazoji, A. Comparative chemical composition of the essential oils of iranian achillea oxyodonta from different ecological regions. J. Appl. Pharm. Sci. 2015, 5, 106-109. [CrossRef]

69. Saeidnia, S.; Yassa, N.; Rezaeipoor, R.; Shafiee, A.; Gohari, A.R.; Kamalinejad, M.; Goodarzi, S. Immunosuppressive principles from achillea talagonica, an endemic species of Iran. DARU J. Fac. Pharm. 2009, 17, 37-41.

70. Saeidnia, S.; Moradi-Afrapoli, F.; Gohari, A.; Malmir, M. Cytotoxic flavonoid from achillea talagonica bioss. J. Med. Plants 2009, 8, 52-56.

71. Gavyar, P.; Amiri, H. Chemical composition of essential oil and antioxidant activity of postia puberula, an endemic species from Iran. Acta Sci. Pol. Hortorum Cultus 2019, 18, 119-128. [CrossRef]

72. Ghassemi, N.; Sajjadi, S.E.; Ghannadi, A.; Shams-Ardakani, M.; Mitra, M. Volatile constituents of a medicinal plant of Iran, echium amoenum Fisch. and C.A. Mey. DARU 2003, 11, 32-33.

73. Mitra, M.; Ghassemi, N.; Sajjadi, S.E.; Ghannadi, A.; Shams-Ardakani, M. Main phenolic compound of petals of echium amoenum Fisch. and C.A. Mey., a famous medicinal plant of Iran. J. Pharm. Sci. 2005, 13, 65-69.

74. Mohammadi, S.; Piri, K.; Dinarvand, M. Antioxidant and antibacterial effects of some medicinal plants of Iran. Int. J. Second. Metab. 2019, 6, 62-78. [CrossRef]

75. Esmaeili, G.; Ghani, A. Antioxidant activity of various fractions extracted from Astragalus adscendens boiss \& haussk as persian Manna. J. Agric. Food Technol. 2017, 8, 12-19.

76. Amiri, M.S.; Joharchi, M.R.; Nadaf, M.; Nasseh, Y. Ethnobotanical knowledge of Astragalus spp.: The world's largest genus of vascular plants. Avicenna J. Phytomed. 2020, 10, 128-142. [PubMed]

77. Hamedi, A.; Zarshenas, M.M.; Sohrabpour, M. Phytochemical assessments of astragalus hamosus pods (Iklil-Ul-Malik). Trends Pharm. Sci. 2016, 2, 77-81.

78. Ghasemian-Yadegari, J.; Hamedeyazdan, S.; Nazemiyeh, H.; Fathiazad, F. Evaluation of phytochemical, antioxidant and antibacterial activity on Astragalus chrysostachys boiss. roots. Iran. J. Pharm. Res. 2019, 18, 1902-1911. [CrossRef] [PubMed]

79. Ziarati, P.; Amirpour, A.; Asgarpanah, J. Chemical composition and nutritive value of astragalus podolobus seeds growing wild in South of Iran. Biomed. Pharmacol. J. 2016, 9, 1117-1125. [CrossRef]

80. Jaimand, K.; Rezaee, M.B.; Mirza, M.; Naderi, M.; Mozaffrian, V.; Azadi, R.; Golipoor, M.; Karimi, S. Essential oil composition of eight hypericum species (Hypericaceae) from Iran: Part II. J. Med. Plants Prod. 2013, 8, 61-68.

81. Jaimand, K.; Rezaee, M.B.; Naderi, M.; Mozaffrian, V.; Azadi, R.; Karimi, S.; Gholipoor, M. Chemical composition of the essential oils of six Hypericum Species (Hypericaceae) from Iran. J. Med. Plants Prod. 2012, 1, 7-11. [CrossRef]

82. Khanavi, M.; Davoodipoor, A.M.; Sadati, S.N.; Ardekani, M.R.S.; Sharifzadeh, M. Antinociceptive effect of some extracts from ajuga Chamaecistus ging. ssp. tomentella (boiss.) rech. f. aerial parts. DARU J. Pharm. Sci. 2014, 22, 56. [CrossRef] [PubMed]

83. Mohammadhosseini, M.; Pazoki, A.; Zamani, H.; Akhlaghi, H. Chemical composition of the essential oil from aerial parts of ajuga chamaecistus ging. subsp. scopria in brackish regions of Iran. J. Essent. Oil-Bear. Plants 2013, 14, 101-105. [CrossRef]

84. Heydari, P.; Yavari, M.; Adibi, P.; Asghari, G.; Ghanadian, S.-M.; Dida, G.; Khamesipour, F. Medicinal properties and active constituents of dracocephalum kotschyi and its significance in Iran: A systematic review. Evid. Based Complement. Altern. Med. 2019, 2019, 9465309. [CrossRef]

85. Ghavam, M. Study of antioxidant activity and some herbal compounds of Dracocephalum kotschyi boiss. in different ages of growth. Biotechnol. Rep. 2019, 25, e00408. [CrossRef]

86. Mirzania, F. Biochemical evaluation of antioxidant activity, phenol and flavonoid contents of dracocephalum kotschyi boiss extracts obtained with different solvents. Health Biotechnol. Biopharma J. 2018, 1, 32-44. [CrossRef]

87. Boroomand, N.; Sadat-Hosseini, M.; Moghbeli, M.; Farajpour, M. Phytochemical components, total phenol and mineral contents and antioxidant activity of six major medicinal plants from rayen, Iran. Nat. Prod. Res. 2017, 32, 564-567. [CrossRef] [PubMed] 
88. Khodaei, M.; Amanzadeh, Y.; Faramarzai, M.-A.; Pirali-Hamedani, M. Chemical analysis and anti-bacterial effect of essential oils from three different species of dracocephalum in Iran. Am. J. Essent. Oil. Nat. Prod. 2018, 6, 31-34.

89. Sonboli, A.; Esmaeili, M.A.; Gholipour, A.; Kanani, M.R. Composition, cytotoxicity and antioxidant activity of the essential oil of dracocephalum surmandinum from Iran. Nat. Prod. Commun. 2010, 5, 1934578X1000500234. [CrossRef]

90. Ebrahimi, S.; Hadian, J.; Sonboli, A. Chemical composition of the essential oil of ziziphora capitata 1. from Iran. J. Essent. Oil Bear. Plants 2013, 12, 678-682. [CrossRef]

91. Jassbi, A.R. Constituents of the essential oil of mentha mozaffariani jamzad. DARU J. Fac. Pharm. 1994, 4, 13-25.

92. Tavakkoli-Khaledi, S.; Asgarpanah, J.; Tavakkoli-Khaledi, S.; Asgarpanah, J. Essential oil chemical composition of mentha mozaffarianii jamzad seeds. J. Mex. Chem. Soc. 2016, 60, 19-22. [CrossRef]

93. Mohammadpour, N.; Emami, S.A.; Asili, J. Identification of volatile oil components of nepeta binaludensis jamzad by gc-ms and 13c-nmr methods and evaluation of its antimicrobial activity. J. Essent. Oil Bear. Plants 2013, 16, 102-107. [CrossRef]

94. Rustaiyan, A.; Nadji, K. Composition of the Essential oils of nepeta ispahanica boiss. and nepeta binaludensis jamzad from iran. Flavour Fragr. J. 1999, 14, 35-37. [CrossRef]

95. Narimani, R.; Moghaddam, M.; Ghasemi Pirbalouti, A.; Mojarab, S. Essential oil composition of seven populations belonging to two nepeta species from northwestern Iran. Int. J. Food Prop. 2017, 20, 2272-2279. [CrossRef]

96. Bhat, A.H.; Alia, A.; Kumar, B.; Mubashir, S. Phytochemical constituents of genus nepeta. J. Chem. $2018,7,7$.

97. Abdoli, P.; Moradkhani, S.; Dastan, D. Comparative analysis of nepeta crispa essential oil composition in flowering and vegetative stages. Am. J. Phytomed. Clin. Ther. 2016, 4, 106-112.

98. Mitra, M.; Asadipour, A.; Amoli, S. Chemical constituents of the essential oil of nepeta depauperata benth. from Iran. DARU J. Pharm. Sci. 2004, 12, 98-100.

99. Bibak, H.; Akbari, A. Essential Oil composition of stems, leaves and flowers of nepeta dschuparensis bornm. from kerman, Iran J. Essent. Oil Bear. Plants 2017, 20,597-600. [CrossRef]

100. Parviz, A.A.; Kambiz, L.; Narjes, K. Chemical composition of the volatile fraction of perovskia abrotanoides and nepeta glomerulosa growing wild in iran by different extraction methods. J. Med. Herbs 2015, 6, 59-64.

101. Yarmohammadi, M.; Talebi, S.; Ghorbani Nohooji, M. Infraspecific variations in essential oil and glandular trichomes in nepeta heliotropifolia. Biodiversitas 2017, 18, 964-970. [CrossRef]

102. Memariani, Z.; Rahimi, A.; Farzaei, M.H.; Nejad, N.Z. Nepeta menthoides boiss. Buhse, an endemic species in iran: A review of traditional uses, phytochemistry and pharmacology. J. Herbmed. Pharmacol. 2019, 8, 194-204. [CrossRef]

103. Shafaghat, A.; Oji, K. Nepetalactone content and antibacterial activity of the essential oils from different parts of Nepeta persica. Nat. Prod. Commun. 2010, 5, 1934578X1000500. [CrossRef]

104. Sajjadi, S.E. Analysis of the essential oil of nepeta sintenisii bornm. from Iran. DARU 2005, 13, 61-64.

105. A Review on Phytochemistry and Pharmacology of Otostegia Persica (Burm. f.) Boiss. J. Med. Plants 2013, 12, 8-18.

106. Sadeghi, Z.; Akaberi, M.; Valizadeh, J. Otostegia persica (Lamiaceae): A review on its ethnopharmacology, phytochemistry, and pharmacology. Avicenna J. Phytomed. 2014, 4, 10.

107. Sharififar, F.; Yassa, N. Antioxidant activity of otostegia persica (Labiatae) and its constituents. Iran. J. Pharm. Res. 2003, 2, 235-239.

108. Tofighi, Z.; Alipour, F.; Yassa, N.; Hadjiakhoondi, A.; Hadavinia, H.; Goodarzi, S.; Golestani, R. Chemical composition and antioxidant activity of otostegia persica essential oil from iran. Int. J. Essent. Oil Ther. 2009, 3, 45-48.

109. Ghorbanpour, M.; Hadian, J.; Hatami, M.; Salehi-Arjomand, H.; Aliahmadi, A. Comparison of chemical compounds and antioxidant and antibacterial properties of various satureja species growing wild in Iran. J. Med. Plants 2016, 15, 58-72.

110. Tepe, B.; Cilkiz, M. A Pharmacological and phytochemical overview on Satureja. Pharm. Biol. 2016, 54, 375-412. [CrossRef]

111. Mohammadpour, G.; Tahmasbpour, R.; Noureini, S.; Bagherpour, G. In vitro antimicrobial and cytotoxicity assays of satureja bakhtiarica and zataria multiflora essential oils. J. Phytomed. Clin. Ther. 2015, 3, 502-511.

112. Hamedi, B.; Mohammadi, Z.; Ghasemi Pirbalouti, A. Antibacterial activity of the essential oils from five endemic herbs (lamiaceae). J. Herb. Drugs Int. J. Med. Herbs 2013, 4, 132-135.

113. Mahboubi, M. Antioxidant and antimicrobial activity of satureja khuzistanica jamzad essential oil, ethanol and aqueous extracts. Biharean Biol. 2017, 12, 37-39.

114. Jassbi, A.R.; Miri, R.; Asadollahi, M.; Javanmardi, N.; Firuzi, O. Cytotoxic, antioxidant and antimicrobial effects of nine species of woundwort (stachys) plants. Pharm. Biol. 2014, 52, 62-67. [CrossRef] [PubMed]

115. Kharazian, N.; Mohammadi, M. Flavonoid patterns and their diversity in Ten stachys L. (Lamiaceae) species from Iran. Prog. Biol. Sci. 2014, 4, 203-218. [CrossRef]

116. Jamzad, M.; Akbari, M.T.; Rustaiyan, A.; Masoudi, S.; Azad, L. Chemical composition of essential oils of three stachys species growing wild in iran: Stachys asterocalyx rech. f., Stachys obtusicrena boiss. and stachys multicaulis benth. J. Essent. Oil Res. 2009, 21, 101-104. [CrossRef]

117. Khanavi, M.; Manayi, A.; Lotfi, M.; Abbasi, R.; Majdzade, M.; Ostad, S.N. Investigation of cytotoxic activity in four stachys species from Iran. Iran. J. Pharm. Res. 2012, 11, 589-593. [CrossRef]

118. Gören, A.C. Use of stachys species (mountain tea) as herbal tea and food. Rec. Nat. Prod. 2014, 8, 71.

119. Sadeghi, H.; Zarezade, V.; Sadeghi, H.; Akbartabar, M.; Jafari Barmak, M.; Azizi, A.; Ghavamizadeh, M.; Mostafazade, M. Anti-Inflammatory activity of stachys pilifera benth. Iran. Red Crescent Med. J. 2014, 16, e19259. [CrossRef] 
120. Farjam, M.H.; Khalili, M.; Rusraiyan, A.; Javidnia, K.; Izadi, S. Biological activity of the N-butanolic extract of stachys pilifera. Afr. J. Microbiol. Res. 2011, 5, 5115-5119. [CrossRef]

121. Ghasemi Pirbalouti, A.; Emami, Z.; Malekpoor, F. An overview on genus thymus. J. Herb. Drugs 2015, 6, 93-100.

122. Mojab, F.; Poursaeed, M.; Mehrgan, H.; Pakdaman, S. Antibacterial activity of thymus daenensis methanolic extract. Pak. J. Pharm. Sci. 2008, 21, 210-213.

123. Khorasany, S.; Azizi, M.H.; Barzegar, M.; Hamidi Esfahani, Z. A study on the chemical composition and antifungal activity of essential oil from thymus caramanicus, thymus daenensis and ziziphora clinopodiaides. Nutr. Food Sci. Res. 2016, 3, 35-42. [CrossRef]

124. Amiri, H. Essential oils composition and antioxidant properties of three thymus species. Evid. Based Complement. Altern. Med. Ecam 2012, 2012, 728065. [CrossRef]

125. Safaei-Ghomi, J.; Meshkatalsadat, M.; Shamaic, S.; Hasheminejad, M.; Hassani, A. Chemical characterization of bioactive volatile molecules of four thymus species using nanoscale injection method. Dig. J. Nanomater. Biostruct. 2009, 4, 835.

126. Ozturk, S. Broad-spectrum antibacterial properties of thymus fallax. Pharm. Biol. 2008, 43, 609-613. [CrossRef]

127. Shayganfar, A.; Sefidkon, F.; Saeedi, I.; Rasouli, M. Aromatic profile of thymus fallax fisch. \& C.A. Mey. essential oil growing wild in Iran. J. Essent. Oil-Bear. Plants 2011, 14, 782-785. [CrossRef]

128. Mobaiyen, H.; Dehghan, G.; Elmi, F.; Talebpour, A.H. The comparison of composition and biological activities in wild and cultivated of thymus kotschyanus essential oils and methanolic extracts from east azarbayjan, Iran. Crescent J. Med. Biol. Sci. 2017, $4,6$.

129. Ahmadi1, R.; Alizadeh, A.; Ketabchi, S. Antimicrobial activity of the essential oil of thymus kotschyanus grown wild in Iran. Int. J. Biosci. 2015, 6, 239-248. [CrossRef]

130. Bakhtiar, Z.; Mirjalili, M.; Sonboli, A. In vitro callus induction and micropropagation of thymus persicus (Lamiaceae), an endangered medicinal plant. Crop Breed. Appl. Biotechnol. 2016, 16, 48-54. [CrossRef]

131. Ghelichnia, H. Essential oil composition of thymus trauvetteri klokov \& desj. at different growing altitudes in mazandaran, Iran. Cercet. Agron. Mold. 2019, 52, 197-205. [CrossRef]

132. Mahmoudvand, H.; Mirbadie, S.R.; Sadooghian, S.; Fasihi Harandi, M.; Jahanbakhsh, S.; Saedi Dezaki, E. Chemical composition and scolicidal activity of Zataria multiflora boiss essential oil. J. Essent. Oil Res. 2016, 29, 42-47. [CrossRef]

133. Rastegar, F.; Moharramipour, S.; Shojai, M.; Abbasipour, H. Chemical composition and insecticidal activity of essential oil of Zataria multiflora boiss. (lamiaceae) against callosobruchus maculatus (F.) (Coleoptera: Bruchidae). IOBC/Wprs Bull. 2011, 69, 281-288.

134. Bidaki, M.; Msc, A. Anti-bacterial effect of Zataria multiflora boiss. essential oil on eight gastrointestinal pathogenic species. Q. Horiz. Med. Sci. 2015, 21, 155-161. [CrossRef]

135. Omidpanah, N.; Valifard, M.; Esmaeili, M.; Yousefi, R.; Moghadam, A. Antioxidant and antibacterial properties of the essential oils of two iranian medicinal plants: Zhumeria majdae and salvia mirzayanii. J. Adv. Med. Sci. Appl. Technol. 2015, 1, 51-60. [CrossRef]

136. Naghibi, F.; Mosaddegh, M.; Mohammadi Motamed, M.; Ghorbani, A. Labiatae family in folk medicine in iran: From ethnobotany to pharmacology. Iran. J. Pharm. Res. 2010, 4, 63-79. [CrossRef]

137. Mohammadhosseini, M. The ethnobotanical, phytochemical and pharmacological properties and medicinal applications of essential oils and extracts of different ziziphora species. Ind. Crops Prod. 2017, 105, 164-192. [CrossRef]

138. Farsam, H.; Amanlou, M.; Amin, G.; Nezamivand-Chegini, G.; Salehi, M.-H. Anatomical and phytochemical study of Lilium ledebourii (Baker) boiss., a rare endemic species in Iran. DARU 2003, 11, 164-170.

139. Amanzadeh, Y.; Hajimehdipoor, H.; Abedi, Z.; Khatamsaz, M. Chemical constituents of Amygdalus spp. oil from Iran. Res. J. Pharmacogn. 2016, 3, 29-33.

140. Jamshidi-Kia, F.; Lorigooini, Z.; Asgary, S.; Saeidi, K. Iranian species of verbascum: A review of botany, phytochemistry, and pharmacological effects. Toxin Rev. 2018, 38, 225-262. [CrossRef]

141. Shakeri, A.R.; Farokh, A. Phytochemical evaluation and antioxidant activity of verbascum sublobatum murb. leaves. Res. J. Pharmacogn. 2015, 2, 43-47. 